\title{
Renormalization Group Analysis of a Simple Hierarchical Fermion Model ${ }^{\star}$
}

\author{
T. C. Dorlas ${ }^{\star \star}$ \\ Institute for Theoretical Physics, Groningen, The Netherlands, and \\ Dublin Institute for Advanced Studies, 10 Burlington Road, Dublin 4, Ireland
}

Received March 20, 1990; in revised form August 10, 1990

\begin{abstract}
A simple hierarchical fermion model is constructed which gives rise to an exact renormalization transformation in a 2-dimensional parameter space. The behaviour of this transformation is studied. It has two hyperbolic fixed points for which the existence of a global critical line is proven. The asymptotic behaviour of the transformation is used to prove the existence of the thermodynamic limit in a certain domain in parameter space. Also the existence of a continuum limit for these theories is investigated using information about the asymptotic renormalization behaviour. It turns out that the "trivial" fixed point gives rise to a twoparameter family of continuum limits corresponding to that part of parameter space where the renormalization trajectories originate at this fixed point. Although the model is not very realistic it serves as a simple example of the application of the renormalization group to proving the existence of the thermodynamic limit and the continuum limit of lattice models. Moreover, it illustrates possible complications that can arise in global renormalization group behaviour, and that might also be present in other models where no global analysis of the renormalization transformation has yet been achieved.
\end{abstract}

\section{Introduction}

Hierarchical models were introduced by Dyson [8] before Wilson [19] formulated his theory of the renormalization group. It was Baker [1] who pointed out the simple renormalization group structure of the model. Actually, Baker's model is different from Dyson's in that it has continuous spins instead of Ising spins. The first mathematical investigation of hierarchical models was carried out by Bleher

* A part of the material here presented was used in the author's thesis

$\star \star$ Present address: University College Swansea, Department of Mathematics and Computer Science, Singleton Park, Swansea, U.K. 
and Sinai [3 and 4]. It was elaborated by Collet and Eckmann [5] and, more recently, by Gawedzki and Kupiainen [11,12]. Here we construct a fermion analogue of Baker's model by replacing the spins with elements of a Grassmann algebra. Thus we obtain a model with a very simple renormalization group structure. It is somewhat like a hierarchical version of the Gross-Neveu model, the renormalization group structure of which was studied by Gawedzki and Kupiainen [14] and Feldman et al. [9]. However, our hierarchical model does not satisfy reflection positivity, so that the continuum limit is not a feasible candidate for a quantum field theory. The renormalization group transformation of our model takes place in a two-dimensional space of coupling parameters $r$ and $g$. It is given by formulas (2.16). The simplicity of the transformation enables us to study the global flow of the transformation.

In the next section the model is introduced and its renormalization transformation derived. In Sect. 3 the main results about the asymptotic behaviour of the transformation are stated and discussed. Theorems 1 and 2 are precise statements about the existence and uniqueness of a global critical line for each of the fixed points $(0,0)$ and $\left(-\frac{4}{3}, \frac{8}{27}\right)$. Theorem 3 is a result about the asymptotic behaviour of points that are not critical. Proofs of these theorems are deferred to Sect. 7. Figure 1 shows a computer picture of the flow of the transformation. In Sect. 4 we use the asymptotic renormalization group behaviour to investigate the existence of a thermodynamic limit. Along the same lines, in Sect. 5, we prove a result about the decay of correlation functions, which is used in the study of the continuum limit in Sect. 6. The existence of a continuum limit is proven for each point on the trajectories receding from the trivial fixed point $(0,0)$. The existence of a continuum limit for points on the line $g=0, r>0$ is the easiest to establish: it is Gaussian. As in the case of the Gross-Neveu model, the other trajectories give rise to non-trivial continuum limits. Section 8 contains some concluding remarks.

\section{The Model}

For a Grassmann algebra $\mathscr{G}_{n}$ with an even number of generators arranged in conjugate pairs $\left\{\psi_{1}, \bar{\psi}_{1}, \ldots, \psi_{n}, \bar{\psi}_{n}\right\}$, there exists an analogue of a Gaussian integral due to Berezin [2]. It is given by the linear functional $\omega_{C}$ on $\mathscr{G}_{n}$ with

$$
\omega_{C}\left(\psi_{i} \bar{\psi}_{j}\right)=\frac{\int \psi_{i} \bar{\psi}_{j} \exp [-\langle\bar{\psi}, B \psi\rangle] d \psi d \bar{\psi}}{\int \exp [-\langle\bar{\psi}, B \psi\rangle] d \psi d \bar{\psi}}=\left(B^{-1}\right)_{i j}=C_{i j}
$$

Here $d \psi d \bar{\psi}$ stands for $d \psi_{1} \ldots d \psi_{n} d \bar{\psi}_{n} \ldots d \bar{\psi}_{1}$ and $\int \cdot d \psi_{i}$ is the usual fermionic integration defined by

$$
\int \psi_{i} d \psi_{i}=1, \quad \int d \psi_{i}=0 .
$$

$B$ is a non-degenerate $n \times n$ matrix and

$$
\langle\bar{\psi}, B \psi\rangle=\sum_{i, j=1}^{n} \bar{\psi}_{i} B_{i j} \psi_{j}
$$

The exponential is given by its (terminating) Taylor expansion.

$\omega_{C}$ satisfies the usual fermionic Wick formulae,

$$
\omega_{C}\left(\psi_{i_{1}} \bar{\psi}_{j_{1}} \psi_{i_{2}} \bar{\psi}_{j_{2}}\right)=C_{i_{1} i_{j}} C_{i_{2} j_{2}}-C_{i_{1} j_{2}} C_{i_{2} j_{1}} \text {, etc. }
$$


For a degenerate matrix $C$ we can still define $\omega_{C}$ by $\omega_{C}\left(\psi_{i} \bar{\psi}_{j}\right)=C_{i j}$, and the Wick formulae.

We now introduce a hierarchical covariance $C$ as follows. We consider a 2-dimensional square lattice $\Lambda_{N} \subset \mathbb{Z}^{2}$ with $\left(2^{N}\right)^{2}$ sites and subdivide it into blocks, blocks of blocks and so forth at each level or scale. The blocks $B_{l}$ at the $l$-th level will contain $2^{2 l}$ lattice sites each. For $x \in \Lambda_{N}$ we denote by $\dot{x} \in \Lambda_{N-1}$ the index of the block $B(\dot{x})$ containing $x$. More generally, $x^{(k)} \in \Lambda_{N-1}$ is defined by $x^{(k-1)} \in B\left(x^{(k)}\right)$. We define a matrix $\Gamma=\left(\Gamma_{x y}\right)_{x, y \in \Lambda_{N}}$ by $\Gamma_{x y}=\left(\Gamma_{\dot{x}}\right)_{x y}$ if $\dot{x}=\dot{y}$ and $\Gamma_{x y}=0$ if $\dot{x} \neq \dot{y} . \Gamma_{\dot{x}}$ is defined on a block $B(\dot{x})$ by

$$
\Gamma_{\dot{x}}=\frac{1}{4}\left(\begin{array}{rrrr}
1 & -1 & 1 & -1 \\
-1 & 1 & -1 & 1 \\
1 & -1 & 1 & -1 \\
-1 & 1 & -1 & 1
\end{array}\right)
$$

with an arbitrary numbering of the sites in a block, fixed once for all. The hierarchical covariance is now defined by the series

$$
\left(C_{N}\right)_{x y}=\sum_{k=0}^{N-1} 2^{-k} \Gamma\left(x^{(k)}, y^{(k)}\right) .
$$

(A slightly different covariance was considered in [6]. Our present choice has the advantage that $M \Gamma=0$, where the operator $M$ is defined by (2.5).) This defines a Gaussian state on the Grassmann algebra generated by the $2 \cdot 2^{2 N}$ spins $\left\{\psi_{x}, \bar{\psi}_{x}\right\}_{x \in \Lambda_{N}}$. Introducing an average spin $(M \psi)_{\dot{x}}$ for each block $B(\dot{x})$ by

$$
(M \psi)_{\dot{x}}=L^{-d+\sigma} \sum_{x \in B(\dot{x})} \psi_{x}
$$

with $d=2, \sigma=\frac{1}{2}, L=2$ and analogously for $\bar{\psi}$, we find for the renormalized covariance (cf. [7]):

$$
\left(C_{N}\right)^{\prime}=M C_{N} M^{t}=C_{N-1} .
$$

Here $M^{t}$ is the transposed matrix.

Before being able to introduce a non-trivial local interaction we have to double the number of spin components so that the corresponding Grassmann algebra $\mathscr{G}_{N}$ now has $4 \cdot 2^{2 N}$ generators. The covariance of the resulting 2-component lattice field becomes $C_{N} \oplus C_{N}$, i.e.

$$
\omega_{C_{N} \oplus C_{N}}\left(\psi_{\alpha x} \bar{\psi}_{\beta y}\right)=\delta_{\alpha \beta}\left(C_{N}\right)_{x y} ; \quad \alpha, \beta=1,2 ; x, y \in \Lambda_{N} .
$$

We consider the general local potentials $V_{N}(\bar{\psi}, \psi)=\sum_{x \in \Lambda_{N}} v\left(\bar{\psi}_{x}, \psi_{x}\right)$ with

$$
v\left(\bar{\psi}_{x}, \psi_{x}\right)=r\left(\bar{\psi}_{1 x} \psi_{1 x}+\bar{\psi}_{2 x} \psi_{2 x}\right)+g \bar{\psi}_{1 x} \psi_{1 x} \bar{\psi}_{2 x} \psi_{2 x} .
$$

In the presence of the potential $V_{N}$ the "expectation value" of a general polynomial $F$ in the fields is given by

$$
\varrho(F)=\frac{\omega_{N}\left(F(\bar{\psi}, \psi) \exp \left[-V_{N}(\bar{\psi}, \psi)\right]\right)}{\omega_{N}\left(\exp \left[-V_{N}(\bar{\psi}, \psi)\right]\right)},
$$

where we have written $\omega_{N}$ instead of $\omega_{C_{N} \oplus C_{N}}$. The renormalized state $\varrho^{\prime}$ is defined on $\mathscr{G}_{N-1}$ by

$$
\varrho^{\prime}(F)=\varrho(F(M \bar{\psi}, M \psi)) .
$$


Due to the fact that

$$
\left(C_{N}\right)_{x y}=\frac{1}{2}\left(C_{N-1}\right)_{\dot{x} \dot{y}}+\Gamma_{x y},
$$

we can split the field $\psi$ into $\psi^{\prime}=M \psi$ and a fluctuation field $\zeta$ with covariance $\Gamma \oplus \Gamma$ so that

$$
\omega_{N}(F(\bar{\psi}, \psi))=\omega_{N-1} \otimes \omega_{\Gamma \oplus \Gamma}\left(F\left(\frac{1}{\sqrt{2}} \bar{\psi}_{x}^{\prime}+\bar{\zeta}_{x}, \frac{1}{\sqrt{2}} \psi_{\dot{x}}^{\prime}+\zeta_{x}\right)\right) .
$$

It then easily follows that

$$
\varrho^{\prime}(F)=\frac{\omega_{N-1}\left(F\left(\bar{\psi}^{\prime}, \psi^{\prime}\right) \exp \left[-V_{N-1}^{\prime}\left(\bar{\psi}^{\prime}, \psi^{\prime}\right)\right]\right)}{\omega_{N-1}\left(\exp \left[-V_{N-1}^{\prime}\left(\bar{\psi}^{\prime}, \psi^{\prime}\right)\right]\right)},
$$

where the effective potential $V_{N-1}^{\prime}$ is also local,

$$
V_{N-1}^{\prime}\left(\bar{\psi}^{\prime}, \psi^{\prime}\right)=\sum_{\dot{x} \in \Lambda_{N-1}} v^{\prime}\left(\bar{\psi}_{x}^{\prime}, \psi_{\dot{x}}^{\prime}\right)
$$

with $v^{\prime}$ given by

$$
\exp \left[-v^{\prime}\left(\bar{\psi}_{\dot{x}}^{\prime}, \psi_{\dot{x}}^{\prime}\right)\right]=\frac{\omega_{\Gamma_{\dot{x}} \oplus \Gamma_{\dot{x}}}\left(\exp \left[-\sum_{x \in \bar{B}(\dot{x})} v\left(\frac{1}{\sqrt{2}} \bar{\psi}_{\dot{x}}^{\prime}+\bar{\zeta}_{x}, \frac{1}{\sqrt{2}} \psi_{x}^{\prime}+\zeta_{x}\right)\right]\right)}{\omega_{\Gamma_{\dot{x} \oplus} \oplus \Gamma_{\dot{x}}}\left(\exp \left[-\sum_{x \in B(\dot{x})} v\left(\bar{\zeta}_{x}, \zeta_{x}\right)\right]\right)}
$$

Remark. In this expression one has to collect the $\zeta$ 's before calculating the expectation with respect to $\omega_{\Gamma_{\dot{x}} \oplus \Gamma_{\dot{x}}}$. Thus, for instance, $\omega_{\Gamma_{0} \oplus \Gamma_{0}}\left(\psi_{\alpha}^{\prime} \zeta_{1} \bar{\psi}_{\beta}^{\prime} \bar{\zeta}_{1}\right)=-\psi_{\alpha}^{\prime} \bar{\psi}_{\beta}^{\prime}$.

The fact that local potentials are conserved under the block-spin transformation is a general property of hierarchical models, much stressed by Gawedzki and Kupiainen $[11,12]$. It is due to the fact that $\Gamma_{x y}=0$ if $\dot{x} \neq \dot{y}$, so that

$$
\omega_{\Gamma \oplus \Gamma}=\bigotimes_{\dot{x} \in A_{N-1}}^{\bigotimes} \omega_{\Gamma_{\dot{x}} \oplus \Gamma_{\dot{x}}} .
$$

Our fermionic hierarchical model has the additional simplifying property that the exponentials in (2.14) break off. Therefore we can calculate the expectations explicitly. Diagonalizing $\Gamma_{x}$ and rewriting the result as an exponential, we obtain

$$
\begin{aligned}
& \omega_{\Gamma_{0} \oplus \Gamma_{0}}\left(\exp \left[-\sum_{x \in B(\dot{x})} v\left(\frac{1}{\sqrt{2}} \bar{\psi}^{\prime}+\bar{\zeta}_{x}, \frac{1}{\sqrt{2}} \psi^{\prime}+\zeta_{x}\right)\right]\right) \\
& =\left[(1+r)^{2}-\frac{1}{4} g\right] \cdot \exp \left[-r^{\prime}\left(\bar{\psi}_{1}^{\prime} \psi_{1}^{\prime}+\bar{\psi}_{2}^{\prime} \psi_{2}^{\prime}\right)-g^{\prime} \bar{\psi}_{1}^{\prime} \psi_{1}^{\prime} \bar{\psi}_{2}^{\prime} \psi_{2}^{\prime}\right]
\end{aligned}
$$

with

$$
\left\{\begin{array}{l}
r^{\prime}=2 r-\frac{1}{2} \frac{g(1+r)}{(1+r)^{2}-g / 4}, \\
g^{\prime}=g\left\{\frac{(1+r)^{2}-g / 2}{(1+r)^{2}-g / 4}\right\}^{2} .
\end{array}\right.
$$

Notice that (2.15) is in fact independent of $\dot{x}$, so that we can omit all indices $\dot{x}$ and write $\Gamma_{0}$ instead of $\Gamma_{\dot{x}}$. 
To derive (2.15) we define

$$
Q=\frac{1}{2}\left(\begin{array}{cccc}
1 & 1 & 1 & 1 \\
1 & 1 & -1 & -1 \\
1 & -1 & 1 & -1 \\
1 & -1 & -1 & 1
\end{array}\right)
$$

and introduce new variables $\eta_{\alpha x}=\left(Q \zeta_{\alpha}\right)_{x}$ satisfying

$$
\omega_{\Gamma_{0} \oplus \Gamma_{0}}\left(\left(Q \zeta_{\alpha}\right)_{x}\left(Q \zeta_{\beta}\right)_{y}\right)=\delta_{\alpha \beta} \delta_{x 3} \delta_{y 3} .
$$

Clearly only the third component $\eta_{\alpha}=\eta_{\alpha 3}$ of $\eta$ matters and we can write

$$
\begin{aligned}
& \omega_{\Gamma_{0} \oplus \Gamma_{0}}\left(\exp \left[-\sum_{x} v\left(\frac{1}{\sqrt{2}} \bar{\psi}^{\prime}+\bar{\zeta}_{x}, \frac{1}{\sqrt{2}} \psi^{\prime}+\zeta_{x}\right)\right]\right) \\
& =\omega_{1}\left(\operatorname { e x p } \left[-2 r \sum_{\alpha=1,2} \sum_{s= \pm 1}\left(\frac{1}{\sqrt{2}} \bar{\psi}_{\alpha}^{\prime}+\frac{1}{2} s \bar{\eta}_{\alpha}\right)\left(\frac{1}{\sqrt{2}} \psi_{\alpha}^{\prime}+\frac{1}{2} s \eta_{\alpha}\right)\right.\right. \\
& -2 g \sum_{s= \pm 1}\left(\frac{1}{\sqrt{2}} \bar{\psi}_{1}^{\prime}+\frac{1}{2} s \bar{\eta}_{1}\right)\left(\frac{1}{\sqrt{2}} \psi_{1}^{\prime}+\frac{1}{2} s \eta_{1}\right) \\
& \left.\left.\times\left(\frac{1}{\sqrt{2}} \bar{\psi}_{2}^{\prime}+\frac{1}{2} s \bar{\eta}_{2}\right)\left(\frac{1}{\sqrt{2}} \psi_{2}^{\prime}+\frac{1}{2} s \eta_{2}\right)\right]\right) \\
& =\exp \left[-2 r\left(\bar{\psi}_{1}^{\prime} \psi_{1}^{\prime}+\bar{\psi}_{2}^{\prime} \psi_{2}^{\prime}\right)-g \bar{\psi}_{1}^{\prime} \psi_{1}^{\prime} \bar{\psi}_{2}^{\prime} \psi_{2}^{\prime}\right] \\
& \times \omega_{1}\left(\operatorname { e x p } \left[-r\left(\bar{\eta}_{1} \eta_{1}+\bar{\eta}_{2} \eta_{2}\right)-\frac{1}{2} g \bar{\psi}_{1}^{\prime} \psi_{1}^{\prime} \bar{\eta}_{2} \eta_{2}\right.\right. \\
& +\frac{1}{2} g \bar{\psi}_{1}^{\prime} \bar{\psi}_{2}^{\prime} \eta_{1} \eta_{2}-\frac{1}{2} g \bar{\psi}_{1}^{\prime} \psi_{2}^{\prime} \eta_{1} \bar{\eta}_{2}-\frac{1}{2} g \psi_{1}^{\prime} \bar{\psi}_{2}^{\prime} \bar{\eta}_{1} \eta_{2} \\
& \left.\left.+\frac{1}{2} g \psi_{1}^{\prime} \psi_{2}^{\prime} \bar{\eta}_{1} \bar{\eta}_{2}-\frac{1}{2} g \bar{\psi}_{2}^{\prime} \psi_{2}^{\prime} \bar{\eta}_{1} \eta_{1}-\frac{1}{4} g \bar{\eta}_{1} \eta_{1} \bar{\eta}_{2} \eta_{2}\right]\right) \\
& =\exp \left[-2 r\left(\bar{\psi}_{1}^{\prime} \psi_{1}^{\prime}+\bar{\psi}_{2}^{\prime} \psi_{2}^{\prime}\right)-g \bar{\psi}_{1}^{\prime} \psi_{1}^{\prime} \bar{\psi}_{2}^{\prime} \psi_{2}^{\prime}\right] \\
& \times\left\{1+2 r+\frac{1}{2} g(1+r)\left(\bar{\psi}_{1}^{\prime} \psi_{1}^{\prime}+\bar{\psi}_{2}^{\prime} \psi_{2}^{\prime}\right)-\frac{1}{4} g+r^{2}+\frac{3}{4} g^{2} \bar{\psi}_{1}^{\prime} \psi_{1}^{\prime} \bar{\psi}_{2}^{\prime} \psi_{2}^{\prime}\right\} \\
& =\left[(1+r)^{2}-\frac{1}{4} g\right] \cdot \exp \left[-r^{\prime}\left(\bar{\psi}_{1}^{\prime} \psi_{1}^{\prime}+\bar{\psi}_{2}^{\prime} \psi_{2}^{\prime}\right)-g^{\prime} \bar{\psi}_{1}^{\prime} \psi_{1}^{\prime} \bar{\psi}_{2}^{\prime} \psi_{2}^{\prime}\right] .
\end{aligned}
$$

From (2.15) it follows that the denominator in (2.14) equals $(1+r)^{2}-\frac{1}{4} g$, so that $v^{\prime}$ has the same form as (2.1) but with $r$ and $g$ replaced by $r^{\prime}$ and $g^{\prime}$ respectively. Apparently, the renormalization transformation for this model is given by a simple explicit transformation $(t, g) \mapsto R(r, g)$ in a 2-dimensional parameter space.

\section{Analysis of the Flow of $R$}

Figure 1 shows a computer picture of the flow of $R$. For clarity successive points under the iteration of $R$ have been connected by straight lines. The parabola is the set of singular points of the transformation. 


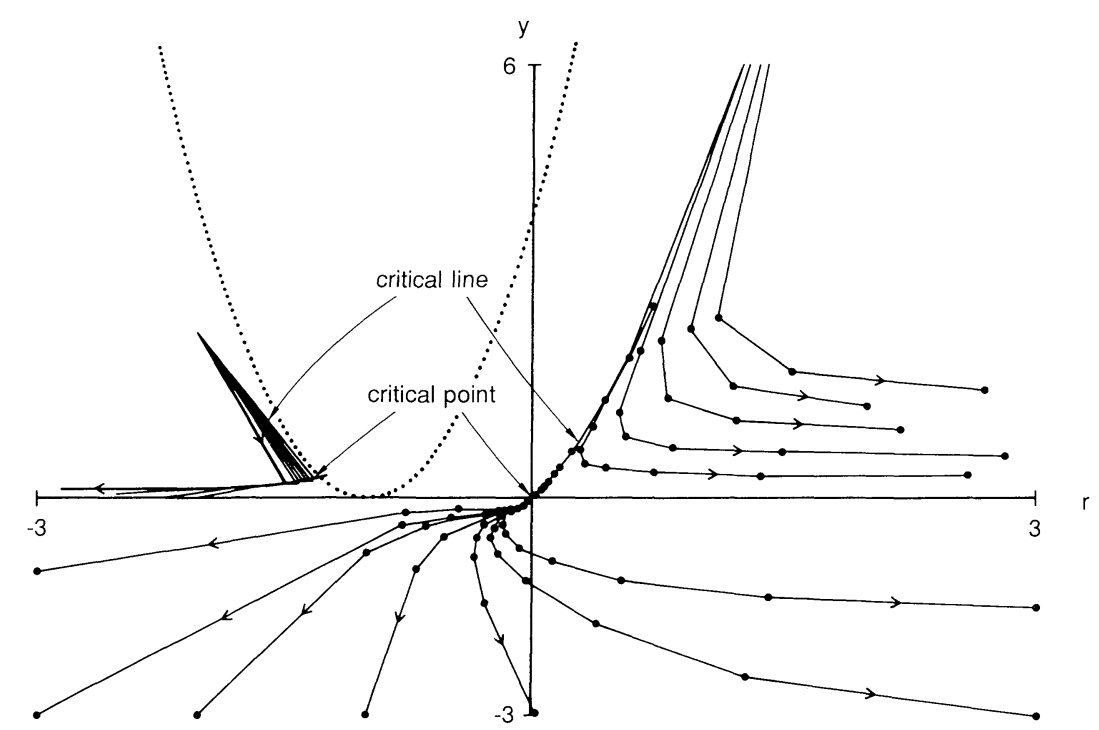

Fig. 1

The fixed points of $R$ are $(0,0)$ and $\left(-\frac{4}{3}, \frac{8}{27}\right)$. By construction the linearized transformation about the origin is

$$
\partial R(0,0)=\left(\begin{array}{cc}
2 & -\frac{1}{2} \\
0 & 1
\end{array}\right)
$$

The $\left(\begin{array}{l}1 \\ 0\end{array}\right)$ eigendirection is repulsive; $\left(\begin{array}{l}1 \\ 2\end{array}\right)$ is a marginal eigendirection. To second order the latter is attracting, which is also clearly visible in Fig. 1. The existence and uniqueness of a critical line in this direction is stated in Theorem 1.

The linearization of $R$ about $\left(-\frac{4}{3}, \frac{8}{27}\right)$ is given by

$$
\partial R\left(-\frac{4}{3}, \frac{8}{27}\right)=\left(\begin{array}{cc}
22 & \frac{27}{2} \\
\frac{64}{3} & 13
\end{array}\right) \text {. }
$$

The eigenvalues are $\lambda_{ \pm}=\frac{35}{2} \pm \frac{3}{2} \sqrt{137} ; \lambda_{+} \approx 35, \lambda_{-} \approx-0.057$. The corresponding eigendirections are $\left(\begin{array}{c}1 \\ -\frac{1}{3} \pm \frac{1}{9} \sqrt{137}\end{array}\right)=\left(\begin{array}{c}1 \\ 0.97\end{array}\right)$ respectively $\left(\begin{array}{c}1 \\ -1.63\end{array}\right)$. The existence and uniqueness of a critical line for this hyperbolic fixed point is stated in Theorem 2. Finally, the asymptotic behaviour of other parameter values $(r, g)$ under the iteration of $R$ is stated in Theorem 3. Unfortunately, we have not been able to prove any rigorous result about the behaviour of points $(r, g)$ with $g>0$ between the two critical lines.

For a precise statement of the existence and uniqueness of a critical line associated with the fixed point $(0,0)$ we define a region $\mathscr{S}\left(g_{0}\right)$ with $g_{0}>0$ as follows. Let the curves $r_{-}(g), r_{+}(g)$, and $r_{0}(g)$ be defined by

$$
\left\{\begin{array}{l}
r_{-}(g)=\frac{1}{2}(\sqrt{1+g}-1) \\
r_{+}(g)=\frac{1}{2}(\sqrt{1+2 g}-1), \\
r_{0}(g)=\sqrt{g / 2}-1
\end{array}\right.
$$


for $g \geqq 0$. Then $\mathscr{S}\left(g_{0}\right)$ is the region bounded by these curves and the line $g=g_{0}$, i.e.

$$
\mathscr{S}\left(g_{0}\right)=\left\{(r, g) \mid 0 \leqq g \leqq g_{0}, \max \left(r_{-}(g), r_{0}(g)\right) \leqq r \leqq r_{+}(g)\right\} .
$$

We shall prove

Theorem 1. For all $g_{0}>0$ there exists a unique critical $r$-value $r_{c}\left(g_{0}\right)>0$ such that

$$
R^{n}\left(r_{c}\left(g_{0}\right), g_{0}\right) \in \mathscr{S}\left(g_{0}\right)
$$

for all $n \geqq 0$, and $R^{n}\left(r_{c}\left(g_{0}\right), g_{0}\right) \rightarrow(0,0)$ as $n \rightarrow \infty$.

The existence of a critical line in the neighbourhood of $(0,0)$ also follows from the Centre Manifold Theorem. We can use it in the form proven by Lanford in [16] (see also [17]) by inverting $R$. The inverse mapping $R^{-1}$ can be calculated explicitly,

$$
\left\{\begin{array}{l}
r=\frac{1}{2} r^{\prime}+\frac{1}{4} \frac{g^{\prime}\left(1+r^{\prime} / 2\right)}{\left(1+r^{\prime} / 2\right)^{2}-g^{\prime} / 2} \\
g=g^{\prime}\left\{\frac{\left(1+r^{\prime} / 2\right)^{2}-g^{\prime} / 4}{\left(1+r^{\prime} / 2\right)^{2}-g^{\prime} / 2}\right\}
\end{array}\right.
$$

The centre manifold is not unique in general. (See Van Strien [18] for a striking counterexample.) Our unique result in Theorem 1 together with the Centre Manifold Theorem imply

Corollary. The critical line $r_{c}(g)$ is $C^{\infty}$ for $g \geqq 0$.

The inverse mapping has also been used to draw the critical line in Fig. 1. It is in fact sufficient to know only a small portion of the critical line to generate the whole line in a finite number of steps. This follows from Lemma 7.2 below together with the fact that, if $g^{\prime}>4 \varepsilon$ with $\varepsilon<\frac{3}{16}$ and $g^{\prime}>r^{\prime}\left(1+r^{\prime}\right)$ then $g>(1+\varepsilon) g^{\prime}$. A small piece of the critical line extending from $g^{\prime}=0$ to $g^{\prime}>4 \varepsilon$ will therefore extend beyond the line $g^{\prime}=2\left(1+\frac{1}{2} r^{\prime}\right)^{2}$ after applying $R^{-1}$ a finite number of times. The next time $R^{-1}$ is applied it extends to infinity. Points above the line $g^{\prime}=2\left(1+\frac{1}{2} r^{\prime}\right)^{2}$ are mapped into the second quadrant, which therefore also contains critical points! Applying $R^{-1}$ sufficiently many times critical points may even return to the neighbourhood of $(0,0)$, which is why Theorem 1 contains the clause $R^{n}\left(r_{c}\left(g_{0}\right), g_{0}\right) \in \mathscr{S}\left(g_{0}\right)$.

An analogous result holds for the fixed point $\left(-\frac{4}{3}, \frac{8}{27}\right)$. We define

$$
\left\{\begin{array}{l}
g_{+}(r)=\frac{8}{3}(1+r)^{2} \\
g_{-}(r)=2(1+r)^{2}+\frac{2}{27}
\end{array},\right.
$$

and

$$
\left\{\begin{array}{l}
\mathscr{T}_{1}=\left\{(r, g) \mid r \leqq-\frac{4}{3}, g_{-}(r) \leqq g \leqq g_{+}(r)\right\} \\
\mathscr{T}_{2}=\left\{(r, g) \mid-\frac{4}{3} \leqq r \leqq r_{0}, g_{+} \leqq g \leqq g_{-}(r)\right\}
\end{array} .\right.
$$

We have put

$$
r_{0}=-\frac{2}{3}-\frac{4}{9} \sqrt{2}
$$

so that $2\left(1+\frac{1}{2} r_{0}\right)^{2}=g_{-}\left(r_{0}\right)$.

Theorem 2. For all $r \leqq r_{0}$ there exists a unique critical value $g_{c}(r)$ such that $R^{n}\left(r, g_{c}(r)\right) \in \mathscr{T}_{1} \cup \mathscr{T}_{2}$ for all $n \geqq 0$ and $R^{n}\left(r, g_{c}(r)\right) \rightarrow\left(-\frac{4}{3}, \frac{8}{27}\right)$ as $n \rightarrow \infty$. 
For the proof of both theorems we make use of a version of the interval argument due to Bleher and Sinai [3]. We need several technical lemmas which are postponed to Sect. 7.

We now state our result about the asymptotic behaviour of other points $(r, g)$ in the plane. This result will prove to be useful for taking the infinite volume limit $N \rightarrow \infty$ and the continuum limit in Sects. 4 and 6 respectively.

Theorem 3. For all $g<0$ there exists a unique value $r_{s}(g)$ of $r$ such that

$$
\begin{gathered}
R^{n}\left(r_{s}(g), g\right) \rightarrow\left(-\frac{2}{3},-\infty\right) \quad \text { as } \quad n \rightarrow \infty, \\
r>r_{s}(g) \Rightarrow R^{n}(r, g) \rightarrow\left(\infty, g_{\infty}(r, g)\right) \text { as } n \rightarrow \infty, \\
r<r_{s}(g) \Rightarrow R^{n}(r, g) \rightarrow\left(-\infty, g_{\infty}(r, g)\right) \text { as } n \rightarrow \infty .
\end{gathered}
$$

where $-\infty<g_{\infty}(r, g)<\infty$.

Furthermore, if $g \geqq 0$ and $r>r_{c}(g)$ then $R^{n}(r, g) \rightarrow\left(\infty, g_{\infty}(r, g)\right)$, and if $r \leqq r_{0}$ and $0 \leqq g<g_{c}(r)$ or $r \leqq-1$ and $0 \leqq g<\frac{8}{3}(1+r)^{2}$ then $R^{n} \rightarrow\left(-\infty, g_{\infty}(r, g)\right)$ as $n \rightarrow \infty$, with $0 \leqq g_{\infty}(r, g)<g$.

We postpone the proof to Sect. 7. Finally we mention a result about the existence of an unstable line for the fixed point $\left(-\frac{4}{3}, \frac{8}{27}\right)$.

Proposition 1.1. For all $r$ between -2 and $-4 / 3$ there exists a unique value $g_{u}(r)$ such that $\left(\tilde{r}_{n}, \tilde{g}_{n}\right) \equiv R^{-n}\left(r, g_{u}(r)\right)$ satisfies $\left(4+\tilde{r}_{n}\right)\left(1+\frac{1}{2} \tilde{r}_{n}\right)^{2} \leqq \tilde{g}_{n} \leqq \frac{8}{3}\left(1+\frac{1}{2} \tilde{r}_{n}\right)^{2}$ for $n \geqq 0$, and $\left(\tilde{r}_{n}, \tilde{g}_{n}\right) \rightarrow\left(-\frac{4}{3}, \frac{8}{27}\right)$ as $n \rightarrow \infty$.

\section{The Infinite Volume Limit}

We can use the renormalization transformation to study the existence of the infinite volume limit $N \rightarrow \infty$ and the existence of the continuum limit (cf. $[11,15]$ ). We shall find that the infinite volume limit exists for the points $(r, g)$ such that $R^{n}(r, g) \rightarrow\left( \pm \infty, g_{\infty}\right)$, and also for the critical points $(r, g)$ associated with the fixed point $(0,0)$, but not for the critical points associated with the fixed point $\left(-\frac{4}{3}, \frac{8}{27}\right)$. An elaboration of the methods used to prove the existence of the thermodynamic limit yields information about the decay of correlation functions. This is shown in Sect. 5. In Sect. 6 this information is used to prove the existence of the continuum limit for points $(r, g)$ on the trajectories receding from the "trivial" fixed point $(0,0)$.

To study the thermodynamic limit $N \rightarrow \infty$ we start by considering the 2-point function $\varrho_{N}\left(\psi_{1 x} \bar{\psi}_{1 y}\right)$ given by (2.9). Assume first that $\dot{x} \neq \dot{y}$. Then we can use the decomposition formula (2.12) to reduce $N$ by 1 . Indeed, by symmetry and the fact that $M \Gamma=0$, it follows that $\varrho_{N}\left(\psi_{1 x} \bar{\psi}_{1 y}\right)=\frac{1}{2} \varrho_{N-1}^{\prime}\left(\psi_{1 \dot{x}}^{\prime} \bar{\psi}_{1 \dot{y}}^{\prime}\right)$. Iterating this relation we find

$$
\varrho_{N}\left(\psi_{1 x} \bar{\psi}_{1 y}\right)=2^{-s} \varrho_{N-s}^{(s)}\left(\psi_{1 x}^{(s)} \bar{\psi}_{1 y}^{(s)}\right)
$$

where $s=s(x, y)$ is the smallest number $s \geqq 0$ such that $y^{(s+1)}=x^{(s+1)}$. We are left with the calculation of $\varrho_{N}\left(\psi_{1 x} \bar{\psi}_{1 y}\right)$ when $\dot{x}=\dot{y}$. Again we apply the decomposition formula (2.12) to find

$$
\varrho_{N}\left(\psi_{1 x} \bar{\psi}_{1 y}\right)=\varrho_{N-1}^{\prime}\left(T\left(\psi_{1 x} \bar{\psi}_{1 y}\right)\right),
$$


where, for a general polynomial $F$ in $\psi$ and $\bar{\psi}$, we define $T F$ by

$$
(T F)\left(\psi^{\prime}, \bar{\psi}^{\prime}\right)=\frac{\omega_{\Gamma \oplus \Gamma}\left(F\left(\frac{1}{\sqrt{2}} \psi^{\prime}+\zeta, \frac{1}{\sqrt{2}} \bar{\psi}^{\prime}+\bar{\zeta}\right) \exp \left[-V\left(\frac{1}{\sqrt{2}} \bar{\psi}^{\prime}+\bar{\zeta}, \frac{1}{\sqrt{2}} \psi^{\prime}+\zeta\right)\right]\right)}{\omega_{\Gamma \oplus \Gamma}\left(\exp \left[-V\left(\frac{1}{\sqrt{2}} \bar{\psi}^{\prime}+\bar{\zeta}, \frac{1}{\sqrt{2}} \psi^{\prime}+\zeta\right)\right]\right)} .
$$

Let us also introduce a block expectation $\langle\cdot\rangle$ by

$$
\langle F(\zeta, \bar{\zeta})\rangle=\frac{\omega_{\Gamma \oplus \Gamma}(F(\zeta, \bar{\zeta}) \exp [-V(\bar{\zeta}, \zeta)])}{\omega_{\Gamma \oplus \Gamma}(\exp [-V(\bar{\zeta}, \zeta)])} .
$$

It is easy to see that this is a product state over blocks, and that, for $x, y$ with $\dot{x}=\dot{y}$,

$$
\left\langle\zeta_{\alpha x} \bar{\zeta}_{\beta y}\right\rangle=\varepsilon_{x} \varepsilon_{y} \delta_{\alpha \beta} c_{1}
$$

with

and

$$
c_{1}=\frac{1}{4} \frac{1+r}{(1+r)^{2}-g / 4}
$$

$$
\varepsilon_{x}=\left\{\begin{array}{lll}
+1, & \text { if } & x=1,3 \\
-1, & \text { if } & x=2,4
\end{array}\right.
$$

i.e. $\varepsilon_{x} \varepsilon_{y}=-1$ if $x$ and $y$ are nearest neighbours, and $=+1$ otherwise (assuming that the points in a block are numbered in a circular way).

Similarly, for $\dot{x}_{1}=\dot{x}_{2}=\dot{y}_{1}=\dot{y}_{2}$,

$$
\left\langle\zeta_{\alpha_{1} x_{1}} \bar{\zeta}_{\beta_{1} y_{1}} \zeta_{\alpha_{2} x_{2}} \bar{\zeta}_{\beta_{2} y_{2}}\right\rangle=\varepsilon_{x_{1}} \varepsilon_{x_{2}} \varepsilon_{y_{1}} \varepsilon_{y_{2}} \gamma\left(\alpha_{1}, \alpha_{2} ; \beta_{1}, \beta_{2}\right) c_{2},
$$

with

and

$$
c_{2}=\frac{1}{16} \frac{1}{(1+r)^{2}-g / 4}
$$

$$
\begin{aligned}
\gamma\left(\alpha_{1}, \alpha_{2} ; \beta_{1}, \beta_{2}\right) & =\left(\delta_{\alpha_{1} \beta_{1}}-\delta_{\alpha_{2} \beta_{2}}\right)\left(1-\delta_{\alpha_{1} \alpha_{2}}\right)\left(1-\delta_{\beta_{1} \beta_{2}}\right) \\
& =\delta_{\alpha_{1} \beta_{1}} \delta_{\alpha_{2} \beta_{2}}-\delta_{\alpha_{1} \beta_{2}} \delta_{\alpha_{2} \beta_{1}} .
\end{aligned}
$$

All other block expectations are zero. We define truncated block expectations

$$
\left\langle F_{1} ; \ldots ; F_{n}\right\rangle=\left\langle\prod_{j=1}^{n} F_{j}\right\rangle_{c}
$$

for monomials $F_{1}, \ldots, F_{n}$ by the usual inductive procedure:

$$
\left\langle F_{1} \ldots F_{n}\right\rangle=\sum_{\left\{I_{l}\right\}_{l=1}^{p}}(-1)^{\pi\left(I_{1}, \ldots, I_{p}\right)} \prod_{l=1}^{p}\left\langle\prod_{i \in I_{l}} F_{i}\right\rangle_{c},
$$

where the sum is over all partitions $\left\{I_{l}\right\}_{l=1}^{p}$ of $\{1, \ldots, n\}$, and $\pi\left(I_{1}, \ldots, I_{p}\right)$ is the number of odd transpositions needed to reorder $(1, \ldots, n)$ according to $I_{1}, \ldots, I_{p}$. A transposition of $i$ and $j$ is called odd if the monomials $F_{i}$ and $F_{j}$ both have odd degree. 
Let us denote a general monomial $F(\psi, \bar{\psi})$ by $\psi_{X}$, where $X$ is a set of pairs $(\alpha, x)$ with $\alpha \in\{1, \overline{1}, 2, \overline{2}\}$. Thus, for example, $\psi_{1 x} \bar{\psi}_{2 y}=\psi_{X}$ with $X=\{(1, x),(\overline{2}, y)\}$. We then have:

Proposition 4.1. The coefficient of $\psi_{Y}^{\prime}$ in the expansion of $T \psi_{X}$ :

is given by

$$
T \psi_{X}=\sum_{Y} 2^{-|Y| / 2} T_{X Y} \psi_{Y}^{\prime}
$$

$$
\begin{aligned}
T_{X Y}= & \sum_{\left\{X_{0} \mid \dot{X}_{0} \subset Y\right\}} \sum_{p=0}^{|Y| X_{0} \mid}(-1)^{p} \sum_{\left\{X_{l}\right\}^{\prime}=1}(-1)^{\sigma\left(X_{0}, \ldots, X_{p}\right)} \\
& \times\left\langle\frac{\partial^{\left|X_{0}\right|} \zeta_{X}}{\partial \zeta_{X_{0}}} ; \frac{\partial^{\left|X_{1}\right|} V}{\partial \zeta_{X_{1}}}(\bar{\zeta}, \zeta) ; \ldots ; \frac{\partial^{\left|X_{p}\right|} V}{\partial \zeta_{X_{p}}}(\bar{\zeta}, \zeta)\right\rangle_{c} .
\end{aligned}
$$

Here the first sum is over all sets $X_{0}$ of pairs $(\alpha, x)$ such that $\dot{X}_{0} \equiv\left\{(\alpha, \dot{x}) \mid(\alpha, x) \in X_{0}\right\} \subset Y$ and the third sum is over all collections of sets $X_{1}, \ldots, X_{p}$ such that $\left\{X_{1}, \ldots, \dot{X}_{p}\right\}$ is a partition of $Y \backslash \dot{X}_{0}$. The sets $X_{0}, \ldots, X_{p}$ are assumed to be ordered according to $Y$ and the differentiation is to be performed in reverse order. $\sigma\left(X_{0}, \ldots, X_{p}\right)$ is the number of transpositions needed to reorder $X$ according to $X_{0}, \ldots, X_{p}$.

Proof. This kind of formula is standard in perturbation theory: see e.g. formula (5.8) in [11]. However, since we are dealing with Grassmann variables here, we have to be careful about reordering factors. This gives rise to factors $(-1)^{\sigma\left(X_{0}, \ldots, X_{p}\right)}$ in the expression (4.12). Notice also that $\frac{\partial^{\left|X_{l}\right|} V}{\partial \zeta_{X_{l}}}=0$ unless all $\zeta_{\alpha x}$ with $(\alpha, x) \in X_{l}$ are situated at the same lattice point $x$. Moreover, looking at the expressions (4.5) and (4.7) for the non-zero block expectations it transpires that changing the lattice point at which $X_{l}$ is situated can only change the sign of the expectation. Since we are summing over all $X_{l}$ it follows that $\left|X_{l}\right|$ must be even. This justifies pulling the derivatives $\frac{\partial^{\left|X_{l}\right|}}{\partial \zeta_{X_{l}}}$ through $F$. It also means that all $\frac{\partial^{\left|X_{l}\right|} V}{\partial \zeta_{X_{l}}}$ are even so that we can restrict the sum to collections $\left\{X_{l}\right\}$ with the same order as $(1, \ldots, n)$ omitting a factor $1 / k !$

Notice that the non-zero terms in (4.12) must satisfy:

1. $X_{0} \subset \operatorname{ind}(F)$ and, in particular, $\left|X_{0}\right| \leqq \operatorname{deg}(F)$, where ind $(F)$ is the index set of $F$, and $\operatorname{deg}(F)$ is the degree of $F$.

2. $\left|X_{l}\right|=2$ for all $l=1,2, \ldots, p$ and the points of $X_{l}$ are equal, because of the factors $\varepsilon_{x}$.

3. $X_{0} \cup X_{1} \cup \ldots \cup X_{p} \subset \bigcup_{x \in \operatorname{supp}(F)} B(\dot{x})$, where $\operatorname{supp}(F)=S$ is the set of points $x$ such that $\psi_{\alpha x}$ occurs in $F(\psi, \bar{\psi})$ for some $\alpha=1, \overline{1}, 2, \overline{2}$. Hence supp $Y \subset S$.

Applying (4.12) to $F(\psi, \bar{\psi})=\psi_{1 x} \bar{\psi}_{1 y}$ we find

$$
T\left(\psi_{1 x} \bar{\psi}_{1 y}\right)=\varepsilon_{x} \varepsilon_{y}\left\{c_{1}+a_{11} \psi_{1 x}^{\prime} \bar{\psi}_{1 x}^{\prime}+a_{12} \psi_{2 x}^{\prime} \bar{\psi}_{2 x}^{\prime}+a_{13} \psi_{1 x}^{\prime} \bar{\psi}_{1 x}^{\prime} \psi_{2 x}^{\prime} \bar{\psi}_{2 x}^{\prime}\right\}
$$

with

$$
\left\{\begin{array}{l}
a_{11}=a_{22}=\frac{1}{2}-2 g\left(c_{2}-c_{1}^{2}\right) \\
a_{12}=a_{21}=2 g c_{1}^{2} \\
a_{13}=a_{23}=8 g^{2} c_{1}\left(c_{1}^{2}-2 c_{2}\right)
\end{array} .\right.
$$


Of course, by symmetry, the same formula (4.13) but with the indices 1 and 2 interchanged, holds for $T\left(\psi_{2 x} \bar{\psi}_{2 y}\right)$ with $\dot{x}=\dot{y}$. Apparently, in order to be able to iterate these equations as in (4.1) we need to include the 4-point function in our consideration. A straightforward calculation yields

$$
T\left(\psi_{1 x} \bar{\psi}_{1 x} \psi_{2 x} \bar{\psi}_{2 x}\right)=c_{2}+a_{31} \psi_{1 \dot{x}}^{\prime} \bar{\psi}_{1 \dot{x}}^{\prime}+a_{32} \psi_{2 \dot{x}}^{\prime} \bar{\psi}_{2 \dot{x}}^{\prime}+a_{33} \psi_{1 \dot{x}}^{\prime} \bar{\psi}_{1 \dot{x}}^{\prime} \psi_{2 x}^{\prime} \bar{\psi}_{2 x}^{\prime},
$$

where

$$
\left\{\begin{array}{l}
a_{31}=a_{32}=\frac{1}{2} c_{1}\left(1+4 g c_{2}\right) \\
a_{33}=\frac{1}{4}+2 g c_{1}^{2}-6 g c_{2}+4 g^{2} c_{2}\left(2 c_{1}^{2}-3 c_{2}\right)
\end{array}\right.
$$

By inserting Eq. (4.13) into (4.2) we have expressed the $\varrho_{N}$-expectation into $\varrho_{N-1}^{\prime}$-expectations. We can do the same with $\varrho_{N}\left(\psi_{1 x} \bar{\psi}_{1 x} \psi_{2 x} \bar{\psi}_{2 x}\right)$ and equation (4.15). In fact the $\varrho_{N}$-expectation of any polynomial can be expressed in terms of a finite number of $\varrho_{N-k}^{(k)}$-expectations of monomials at a single point $x^{(k)}$. Thus, in order to prove the existence of the thermodynamic limit it suffices to consider the monomials at a single point $x$ :

Theorem 4. Let $\mathscr{D}$ be the set of points $(r, g)$ satisfying $g<0$ or $\left(g>0\right.$ and $\left.r \geqq r_{c}(g)\right)$ or $\left(r \leqq r_{0}\right.$ and $\left.g<g_{c}(r)\right)$ or $\left(r_{0}<r<-1\right.$ and $\left.g<\frac{8}{3}(1+r)^{2}\right)$, where $r_{0}$ is defined by (3.9) and $r_{c}(g)$ and $g_{c}(r)$ have been defined in Theorems 2 and 3. Define the state $\varrho_{N}$ on the Grassmann algebra $\mathscr{G}\left(E_{N}\right)$ over $E_{N}=\left(\mathbb{R}^{2}\right)^{\Lambda_{N}}$ by (2.9). Then the thermodynamic limit exists if $(r, g) \in \mathscr{D}$ in the sense that $\varrho(F)=\lim _{N \rightarrow \infty} \varrho_{N}(F)$ exists for all polynomials $F$ in the
fields.

Proof. It is sufficient to consider the cases $F=\psi_{1 x} \bar{\psi}_{1 x}, F=\psi_{2 x} \bar{\psi}_{2 x}$, and

$$
F=\psi_{1 x} \bar{\psi}_{1 x} \psi_{2 x} \bar{\psi}_{2 x}
$$

By symmetry all other single point expectations vanish. Also,

$$
\varrho_{N}\left(\psi_{1 x} \bar{\psi}_{1 x}\right)=\varrho_{N}\left(\psi_{2 x} \bar{\psi}_{2 x}\right)
$$

Writing

$$
\left\{\begin{array}{l}
u_{1}^{(N)}=u_{2}^{(N)}=\varrho_{N}\left(\psi_{1 x} \bar{\psi}_{1 x}\right) \text { and } \\
u_{3}^{(N)}=\varrho_{N}\left(\psi_{1 x} \bar{\psi}_{1 x} \psi_{2 x} \bar{\psi}_{2 x}\right)
\end{array}\right.
$$

and defining the matrices

$$
A^{(n)}=\left(\begin{array}{lll}
a_{11}^{(n)} & a_{12}^{(n)} & a_{13}^{(n)} \\
a_{21}^{(n)} & a_{22}^{(n)} & a_{23}^{(n)} \\
a_{31}^{(n)} & a_{32}^{(n)} & a_{33}^{(n)}
\end{array}\right),
$$

where the $a_{i j}^{(n)}$ are given by (4.14) and (4.16) with $r$ and $g$ replaced by $r^{(n)}$ and $g^{(n)}$, we can rewrite $(4.13)$ and $(4.15)$ in the form of a vector equation:

$\mathbf{u}^{(N)}=\mathbf{c}^{(0)}+A^{(0)} \mathbf{u}^{(N-1)}$, and more generally,

$$
\mathbf{u}^{(N-n)}=\mathrm{c}^{(n)}+A^{(n)} \mathbf{u}^{(N-n-1)}
$$

Iterating this equation we obtain

$$
\mathbf{u}^{(N)}=\sum_{n=0}^{N} A^{(0)} \ldots A^{(n-1)} \mathbf{c}^{(n)} .
$$


We used the fact that $\mathbf{u}^{(0)}=\mathbf{c}^{(N)}$; the $n=0$-term is simply $\mathbf{c}^{(0)}$.

We now want to study the convergence of $(4.20)$ as $N \rightarrow \infty$. We use the following lemma:

Lemma 4.1. Suppose that $\limsup _{n \rightarrow \infty}\left\|A^{(n)}\right\|<1$ and that $\left\|\mathbf{c}^{(n)}\right\|$ is bounded.

Then $\sum_{n=0}^{\infty} A^{(0)} \ldots A^{(n-1)} \mathbf{c}^{(n)}$ converges.

Now, for points $(r, g)$ such that $R^{n}(r, g) \rightarrow\left( \pm \infty, g_{\infty}\right)$ we have

and

$$
A^{(n)} \rightarrow\left(\begin{array}{ccc}
1 / 2 & 0 & 0 \\
0 & 1 / 2 & 0 \\
0 & 0 & 1 / 4
\end{array}\right) \text { as } n \rightarrow \infty
$$

$$
c_{1}^{(n)}=\frac{1}{4} \frac{1+r^{(n)}}{\left(1+r^{(n)}\right)^{2}-g^{(n)} / 4} \rightarrow 0 \quad \text { and } \quad c_{2}^{(n)}=\frac{1}{16} \frac{1}{\left(1+r^{(n)}\right)^{2}-g^{(n)} / 4} \rightarrow 0,
$$

so that the thermodynamic limit exists. For points $(r, g)$ such that $\left(r^{(n)}, g^{(n)}\right) \rightarrow(0,0)$, i.e. $r=r_{c}(g)$, we have

$$
A^{(n)} \rightarrow\left(\begin{array}{ccc}
1 / 2 & 0 & 0 \\
0 & 1 / 2 & 0 \\
1 / 8 & 1 / 8 & 1 / 4
\end{array}\right) \quad \text { and } \quad \mathbf{c}^{(n)} \rightarrow\left(\begin{array}{c}
1 / 4 \\
1 / 4 \\
1 / 8
\end{array}\right)
$$

so that, again, $\lim _{N \rightarrow \infty} \mathbf{u}^{(N)}$ exists.

Next consider the points $(r, g)$ with $r=r_{s}(g)$, so that $R^{n}(r, g) \rightarrow\left(-\frac{2}{3},-\infty\right)$. In that case

$$
A^{(n)} \rightarrow\left(\begin{array}{ccc}
1 & 0 & -4 / 3 \\
0 & 1 & -4 / 3 \\
0 & 0 & 1
\end{array}\right) \text { and } \mathbf{c}^{(n)} \rightarrow\left(\begin{array}{l}
0 \\
0 \\
0
\end{array}\right)
$$

and Lemma 4.1 does not apply. Instead we can use

Lemma 4.2. Suppose that $\frac{\left\|c^{(n+1)}\right\|}{\left\|c^{(n)}\right\|} \rightarrow \delta>0$ and $\limsup _{n \rightarrow \infty}\left\|A^{(n)}\right\|<\delta^{-1}$.

Then $\sum_{n=0}^{\infty} A^{(0)} \ldots A^{(n-1)} \mathbf{c}^{(n)}$ converges absolutely.

In the present case, $g^{(n+1)} \sim 4 g^{(n)}$, so that $\mathbf{c}^{(n+1)} \sim \frac{1}{4} \mathbf{c}^{(n)}$, and $\left\|A^{(n)}\right\| \rightarrow\left\|A_{\infty}\right\|$ $=2.15<4$. It follows that $\sum_{n=0} A^{(0)} \ldots A^{(n-1)} \mathbf{c}^{(n)}$ still converges.

Finally, we consider the points $(r, g)$ on the critical line belonging to the fixed point $\left(-\frac{4}{3}, \frac{8}{27}\right)$, i.e. $g=g_{c}(r)$. We have

$$
A^{(n)} \rightarrow\left(\begin{array}{ccc}
5 / 2 & 3 & -8 / 3 \\
3 & 5 / 2 & -8 / 3 \\
-27 / 8 & -27 / 8 & 13 / 4
\end{array}\right) \quad \text { and } \quad \mathbf{c}^{(n)} \rightarrow\left(\begin{array}{c}
-9 / 4 \\
-9 / 4 \\
27 / 16
\end{array}\right)
$$

and $A^{(\infty)}$ has three eigenvalues: $-\frac{1}{2}$ and $\lambda_{+}=\frac{35}{8} \pm \frac{3}{8} \sqrt{137}$. Since $\left(-\frac{9}{4}, \frac{27}{16}\right)$ has a component in the expanding right-eigendirection, $\left\|\left(A^{(\infty)}\right)^{k} \mathbf{c}^{(\infty)}\right\| \rightarrow \infty$, and the thermodynamic limit does not exist. 


\section{Decay of Correlation Functions}

By an extension of the methods used to prove the existence of the thermodynamic limit we can obtain information about the decay of correlation functions. We shall do this in the region $g<0$ which is relevant for the continuum limit.

We need a few definitions:

Given a point $(r, g)$ with $g<0$ we define the trajectory $\mathscr{T}(r, g)$ as the set of points $R^{n}(r, g)$ with $n=\ldots,-2,-1,0,1,2, \ldots$ The (truncated) correlation functions

$$
\begin{aligned}
\varrho^{T}\left(\psi_{\alpha_{1} x_{1}} \bar{\psi}_{\beta_{1} y_{1}} \ldots \psi_{\alpha_{n} x_{n}} \bar{\psi}_{\beta_{n} y_{n}}\right) & =\varrho^{T}\left(\psi_{\alpha_{1} x_{1}} \ldots \psi_{\alpha_{n} x_{n}} \bar{\psi}_{\beta_{n} y_{n}} \ldots \bar{\psi}_{\beta_{1} y_{1}}\right) \\
& =\varrho^{T}\left(\prod_{i=1}^{\vec{n}} \psi_{\alpha_{i} x_{i}} \prod_{j=1}^{\overleftarrow{n}} \bar{\psi}_{\beta_{j} y_{j}}\right)
\end{aligned}
$$

are defined inductively as in (4.10):

$$
\varrho\left(\psi_{X} \bar{\psi}_{Y}\right)=\sum_{\left\{X_{k}\right\}_{k=1}^{p}} \sum_{\left\{Y_{k}\right\}_{k=1}^{p}:\left|Y_{k}\right|=\left|X_{k}\right|}(-1)^{\pi\left(\left\{X_{k}\right\}\right)+\pi\left(\left\{Y_{k}\right\}\right)} \prod_{k=1}^{p} \varrho^{T}\left(\psi_{X_{k}} \bar{\psi}_{Y_{k}}\right),
$$

where $\left\{X_{k}\right\}$ and $\left\{Y_{k}\right\}$ are partitions of the sets $X=\left\{\left(\alpha_{i}, x_{i}\right)\right\}_{i=1}^{n}$ and $Y=\left\{\left(\beta_{j}, y_{j}\right)\right\}_{j=1}^{n}$ respectively.

For two sequences of points $\mathbf{x}=\left(x_{1}, \ldots, x_{n}\right)$ and $\mathbf{y}=\left(y_{1}, \ldots, y_{n}\right)$ we define a quantity $l(\mathbf{x}, \mathbf{y})$ as follows.

Let $\mathscr{S}(\mathbf{x}, \mathbf{y})$ be the set of permutations $\sigma \in \mathscr{S}_{n}$ of $(1, \ldots, n)$ such that every block $B_{s}$ at any level $s$ containing at least one point $x_{i}$ or $y_{j}$ but not all $x_{i}$ and all $y_{j}$ is connected to another block $B_{s}^{\prime}$. Here two level-s blocks $B_{s}$ and $B_{s}^{\prime}$ are called connected if there exists an $i=1, \ldots, n$ such that either $x_{i} \in B_{s}$ and $y_{\sigma(i)} \in B_{s}^{\prime}$ or $x_{i} \in B_{s}^{\prime}$ and $y_{\sigma(i)} \in B_{s}$.

Next we define

$$
l(\mathbf{x}, \mathbf{y})=\min _{\sigma \in \mathscr{S}(\mathbf{x}, \mathbf{y})} \sum_{i=1}^{n} s\left(x_{i}, y_{\sigma(i)}\right)
$$

if $\mathscr{S}(\mathbf{x}, \mathbf{y}) \neq \emptyset$ and $l(\mathbf{x}, \mathbf{y})=\infty$ otherwise.

The main theorem in this section is:

Theorem 5. Let $(r, g)$ be a point in the lower half-plane $(g<0)$ such that $R^{n}(r, g)$ $\rightarrow\left( \pm \infty, g_{\infty}\right)$. For all $n \geqq 1$ there exists a constant $C_{n}(r, g)$ depending only on $n$ and the trajectory $\mathscr{T}(r, g)$, such that, for any set of $2 n$ points $x_{1}, \ldots, x_{n}, y_{1}, \ldots, y_{n}$ and indices $\alpha_{1}, \ldots, \alpha_{n}, \beta_{1}, \ldots, \beta_{n} \in\{1,2\}$,

$$
\left|\varrho^{T}\left(\psi_{\alpha_{1} x_{1}} \bar{\psi}_{\beta_{1} y_{1}} \ldots \psi_{\alpha_{n} x_{n}} \bar{\psi}_{\beta_{n} y_{n}}\right)\right| \leqq C_{n}(r, g) 2^{-l(\mathbf{x}, \mathbf{y})}
$$

with $\mathbf{x}=\left(x_{1}, \ldots, x_{n}\right)$ and $\mathbf{y}=\left(y_{1}, \ldots, y_{n}\right)$.

We shall prove this theorem by inductive application of the renormalization transformation. However, unlike the proof of Theorem 4, we do not have a nice iteration formula for $\varrho^{T}$ as we did for $\varrho$ in (4.2). We therefore replace the truncated expectation (5.1) by partially truncated expectations of the form

$$
\varrho\left(\prod_{k=1}^{m} ; F_{k}\right)=\varrho\left(F_{1} ; F_{2} ; \ldots ; F_{m}\right)
$$

where each $F_{k}$ is a polynomial concentrated at a single point $z_{k}$, i.e. $\operatorname{supp}\left(F_{k}\right)=\left\{z_{k}\right\}$, and $z_{k} \neq z_{k^{\prime}}$ if $k \neq k^{\prime}$. In fact we can subdivide the polynomials $F_{k}$ into classes which 
are invariant under renormalization, as follows. For an arbitrary monomial

$$
F=\psi_{\alpha_{1} x_{1}} \ldots \psi_{\alpha_{n} x_{n}} \bar{\psi}_{\beta_{m} y_{m}} \ldots \bar{\psi}_{\beta_{1} y_{1}},
$$

we introduce "charges" $q_{\alpha}(F)(\alpha=1,2)$ by

$$
q_{\alpha}(F)=\#\left\{i \mid \alpha_{i}=\alpha\right\}-\#\left\{j \mid \beta_{j}=\alpha\right\} .
$$

Next we observe

Lemma 5.1. If $F$ is a monomial then $T(F)$ given by (4.11) is a charge-homogeneous polynomial, and $q_{\alpha}(T(F))=q_{\alpha}(F)$.

Proof. Clearly, for any polynomial $G$ in $\zeta$ and $\bar{\zeta},\langle G\rangle=0$ unless $q_{\alpha}(G)=0(\alpha=1,2)$. It follows that, in formula (4.12),

$$
q_{\alpha}(F)-q_{\alpha}\left(\psi_{X_{0}}\right)-\sum_{j=1}^{p} q_{\alpha}\left(\psi_{X_{j}}\right)=0 \quad \text { if } \quad T_{X Y} \neq 0 .
$$

But $Y=\bigcup_{j=0}^{p} \dot{X}_{j}$ so that

$$
q_{\alpha}\left(\psi_{Y}^{\prime}\right)=q_{\alpha}\left(\psi_{X_{0}}\right)+\sum_{k=1}^{p} q_{\alpha}\left(\psi_{X_{k}}\right)=q_{\alpha}(F) .
$$

We now restrict our consideration to polynomials of the form $F=\prod_{k=1}^{m} F_{k}$, where the polynomials $F_{k}$ are concentrated at distinct points $z_{k}$ and each $F_{k}$ is charge-homogeneous, i.e. it belongs to one of the following classes:

Table 1

\begin{tabular}{llll}
\hline$q_{1}=+1$ & $q_{2}=+1$ & $F=f \psi_{1} \psi_{2}$ & $d=0$ \\
$q_{1}=-1$ & $q_{2}=-1$ & $F=f \bar{\psi}_{1} \bar{\psi}_{2}$ & $d=0$ \\
$q_{1}=+1$ & $q_{2}=0$ & $F=f_{1} \psi_{1}+f_{2} \psi_{1} \psi_{2} \bar{\psi}_{2}$ & $d=1$ \\
$q_{1}=0$ & $q_{2}=+1$ & $F=f_{1} \psi_{2}+f_{2} \psi_{1} \bar{\psi}_{1} \psi_{2}$ & $d=1$ \\
$q_{1}=-1$ & $q_{2}=0$ & $F=f_{1} \bar{\psi}_{1}+f_{2} \bar{\psi}_{1} \psi_{2} \bar{\psi}_{2}$ & $d=1$ \\
$q_{1}=0$ & $q_{2}=-1$ & $F=f_{1} \bar{\psi}_{2}+f_{2} \psi_{1} \bar{\psi}_{1} \bar{\psi}_{2}$ & $d=1$ \\
$q_{1}=0$ & $q_{2}=0$ & $F=f_{0}+f_{1} \psi_{1} \bar{\psi}_{1}+f_{2} \psi_{2} \bar{\psi}_{2}+f_{3} \psi_{1} \bar{\psi}_{1} \psi_{2} \bar{\psi}_{2}$ & $d=0$ \\
$q_{1}=-1$ & $q_{2}=+1$ & $F=f \bar{\psi}_{1} \psi_{2}$ & $d=0$ \\
$q_{1}=+1$ & $q_{2}=-1$ & $F=f \psi_{1} \bar{\psi}_{2}$ & $d=0$ \\
\hline
\end{tabular}

The third column in this table shows the general form of a polynomial $F_{k}$ belonging to this class. The $f$ 's are coefficients, $\psi_{1}$ stands for $\psi_{1 z_{k}}$ etc. The number $d$ is defined in formula (5.12).

The collection of polynomials $F=\prod_{k=1}^{m} F_{k}$ described above we shall denote by $\mathscr{F}$. For $F \in \mathscr{F}$ we write $\varrho(; F)$ for $\varrho\left(\prod_{k=1}^{m} ; F_{k}\right)$. It is defined analogous to
$\left\langle F_{1} ; \ldots ; F_{n}\right\rangle_{c}$ in Sect. 4 .

Instead of Theorem 5 we shall prove an analogous result for the expectations $\varrho(; F)$, from which Theorem 5 then follows. In order to formulate this result we 
need to extend the definition of $l(\mathbf{x}, \mathbf{y})$ to polynomials of this form. This is possible because of the following fact.

Lemma 5.2. Suppose that, for two sequences of points $\mathbf{x}=\left(x_{1}, \ldots, x_{n}\right)$ and $\mathbf{y}=\left(y_{1}, \ldots, y_{n}\right)$ the following holds:

$\dot{x}_{n}=\dot{y}_{n}$ and there exists $i \neq n$ such that $\dot{x}_{i}=\dot{x}_{n}$ or $\dot{y}_{i}=\dot{y}_{n}$ (or both). Then $l(\mathbf{x}, \mathbf{y})$ $=l\left(\mathbf{x}^{\prime}, \mathbf{y}^{\prime}\right)$, where $\mathbf{x}^{\prime}=\left(x_{1}, \ldots, x_{n-1}\right)$ and $\mathbf{y}^{\prime}=\left(y_{1}, \ldots, y_{n-1}\right)$.

Proof. Suppose that $\sigma \in \mathscr{S}\left(\mathbf{x}^{\prime}, \mathbf{y}^{\prime}\right)$. Then we can define $\tilde{\sigma} \in \mathscr{S}(\mathbf{x}, \mathbf{y})$ by $\tilde{\sigma}(i)=\sigma(i)$ for $i=1, \ldots, n-1$, and $\tilde{\sigma}(n)=n$. Obviously $l(\tilde{\sigma})=l^{\prime}(\sigma)$ if we put

$$
l^{\prime}(\sigma)=\sum_{i=1}^{n-1} s\left(x_{i}, y_{\sigma(i)}\right) \quad \text { and } \quad l(\tilde{\sigma})=\sum_{i=1}^{n} s\left(x_{i}, y_{\tilde{\sigma}(i)}\right)
$$

Conversely, for $\tau \in \mathscr{S}(\mathbf{x}, \mathbf{y})$ we shall presently construct $\sigma \in \mathscr{S}\left(\mathbf{x}^{\prime}, \mathbf{y}^{\prime}\right)$ with $l^{\prime}(\sigma)$ $\leqq l(\tau)$. That completes the proof of the lemma. We may assume that, for some $i \neq n$, $\dot{x}_{i}=\dot{x}_{n}=\dot{y}_{n}$. (The case $\dot{y}_{i}=\dot{x}_{n}=\dot{y}_{n}$ is similar.) There are five separate cases to be considered:

(i) $\dot{x}_{n}=\dot{y}_{\tau(n)}$ or $\dot{x}_{n}=\dot{x}_{\tau-1(n)}$ or $\dot{x}_{i}=\dot{y}_{\tau(i)}$.

(ii) $\dot{x}_{\tau-1(n)}=\dot{y}_{\tau(n)}=\dot{y}_{\tau(i)} \neq \dot{x}_{n}$.

(iii) $\dot{x}_{i} \neq \dot{y}_{\tau(n)}=\dot{y}_{\tau(i)} \neq \dot{x}_{\tau-1(n)} \neq \dot{x}_{i}$ or $\dot{x}_{i} \neq \dot{x}_{\tau-1(n)}=\dot{y}_{\tau(i)} \neq \dot{y}_{\tau(n)} \neq \dot{x}_{i}$.

(iv) $\dot{x}_{i} \neq \dot{x}_{\tau-1(n)}=\dot{y}_{\tau(n)} \neq \dot{y}_{\tau(i)} \neq \dot{x}_{i}$.

(v) $\dot{x}_{i}=\dot{x}_{n}=\dot{y}_{n}$ and $\dot{x}_{\tau^{-1}(n)}$ and $\dot{y}_{\tau(n)}$ and $\dot{y}_{\tau(i)}$ all different.

We simply state the definition of $\sigma$ in the various cases. The verification of the fact that $\sigma \in \mathscr{S}\left(\mathbf{x}^{\prime}, \mathbf{y}^{\prime}\right)$ and $l^{\prime}(\sigma) \leqq l(\tau)$ is simple and will be omitted.

Remark. Notice that the connectivity of $s=0$-blocks, i.e. points, need not be checked because if there is a point $y_{\sigma(j)}=x_{j}$ then the level-1 block containing that point contains another point $x_{k}$ connected outside the block; we can then simply modify $\sigma$ as follows: $\sigma^{\prime}(i)=\sigma(j)$ and $\sigma^{\prime}(j)=\sigma(i)$.

In case (i), if $\dot{y}_{\tau(n)}=\dot{x}_{n}$ but $\tau(n) \neq n$ we can put $\sigma\left(\tau^{-1}(n)\right)=\tau(n)$. If $\dot{y}_{\tau(i)}=\dot{x}_{n}$ then we put $\sigma(i)=\tau(n)$ and $\sigma\left(\tau^{-1}(n)\right)=\tau(i)$ and if $\dot{x}_{\tau^{-1}(n)}=\dot{x}_{n}$ we put $\sigma\left(\tau^{-1}(n)\right)=\tau(n)$. In case (ii) and (iii) we put $\sigma\left(\tau^{-1}(n)\right)=\tau(n)$. In case (iv) we define $\sigma(i)=\tau(n)$ and $\sigma\left(\tau^{-1}(n)\right)$ $=\tau(i)$. In case $(\mathrm{v})$ we must distinguish the following two possibilities:

(a) $s\left(x_{i}, y_{\tau(n)}\right)+s\left(x_{\tau^{-1}(n)}, y_{\tau(i)}\right) \leqq s\left(x_{i}, y_{\tau(i)}\right)+s\left(x_{\tau^{-1}(n)}, y_{\tau(n)}\right)$, and

(b) $s\left(x_{i}, y_{\tau(n)}\right)+s\left(x_{\tau-1}^{-1(n)}, y_{\tau(i)}\right)>s\left(x_{i}, y_{\tau(i)}\right)+s\left(x_{\tau-1}(n), y_{\tau(n)}\right)$.

In the first case we put $\sigma(i)=\tau(i)$ and $\sigma\left(\tau^{-1}(n)\right)=\tau(n)$; in the second case we put $\sigma(i)=\tau(n)$ and $\sigma\left(\tau^{-1}(n)\right)=\tau(i)$. From this lemma it follows that, for a polynomial $F=\prod_{k=1}^{m} F_{k}$ in the class $\mathscr{F}$, we
can define

$$
l\left(\prod_{k=1}^{m} F_{k}\right)=l\left(\bigcup_{k=1}^{m} X_{k}\right),
$$

where $\psi_{X_{k}}$ is one of the monomials of $F_{k}\left(X_{k} \subset\left\{\left(1, z_{k}\right),\left(\overline{1}, z_{k}\right),\left(2, z_{k}\right),\left(\overline{2}, z_{k}\right)\right\}\right)$. We can now state the result for $\varrho(; F)$ from which Theorem 5 follows:

Lemma 5.3. If $F=\prod_{k=1}^{m} F_{k}$ is a polynomial in the class $\mathscr{F}$ then there exists a constant $\widetilde{C}_{m}(r, g)$ depending only on $m$ and the trajectory $\mathscr{T}(r, g)$ such that

$$
|\varrho(; F)| \leqq \widetilde{C}_{m}(r, g) \prod_{k=1}^{m}\left\|F_{k}\right\| 2^{-l(F)}
$$


Proof of Theorem 5 given Lemma 5.3. We use the following relation:

Lemma 5.4. Let $F_{k}=\psi_{X_{k}} \bar{\psi}_{Y_{k}}$. Then

$$
\varrho(; F)=\sum_{\left\{X_{l}^{\prime}\right\}_{l=1}^{p}}^{*} \sum_{\left\{Y_{l}^{\prime}\right\}_{l=1}^{p}}^{*}(-1)^{\pi\left(\left\{X_{l}^{\prime}\right\}\right)+\pi\left(\left\{Y_{l}^{\prime}\right\}\right)} \prod_{l=1}^{p} \varrho^{T}\left(\psi_{X_{l}^{\prime}} \bar{\psi}_{Y_{l}^{\prime}}\right),
$$

where the sum is taken over all partitions $\left\{X_{l}^{\prime}\right\}_{l=1}^{p}$ and $\left\{Y_{l}^{\prime}\right\}_{l=1}^{p}$ of $\bigcup_{k=1}^{m} X_{k}$ and $\bigcup_{k=1}^{m} Y_{k}$ respectively, such that $\left|X_{l}^{\prime}\right|=\left|Y_{l}^{\prime}\right|$ and for each pair $\left(k, k^{\prime}\right)$ with $k, k^{\prime} \in\{1,2, \ldots, m\}$ there exists a sequence of "links" $\left(k_{i}, k_{i+1}\right)(i=1, \ldots, r-1)$ such that $k_{1}=k, k_{r}=k^{\prime}$ and for each $i=1, \ldots, r-1$ there exists $l_{i} \in\{1, \ldots, p\}$ such that $X_{k_{i}} \cap X_{l_{i}}^{\prime} \neq \emptyset$ and $Y_{k_{i}+1} \cap Y_{l_{i}}^{\prime} \neq \emptyset$ or $X_{k_{i+1}} \cap X_{l_{i}}^{\prime} \neq \emptyset$ and $Y_{k_{i}} \cap Y_{l_{i}}^{\prime} \neq \emptyset$.

Now assume that Lemma 5.3 has been proven. We can then make the following rough estimate by moving all the terms in (5.9) with $p>1$ to the left-hand side of the equation:

$$
\begin{aligned}
& \left|\varrho^{T}\left(\psi_{\alpha_{1} x_{1}} \ldots \psi_{\alpha_{n} x_{n}} \bar{\psi}_{\beta_{n} y_{n}} \ldots \bar{\psi}_{\beta_{1} y_{1}}\right)\right| \leqq \widetilde{C}_{m}(r, g) 2^{-l(\mathbf{x}, \mathbf{y})}
\end{aligned}
$$

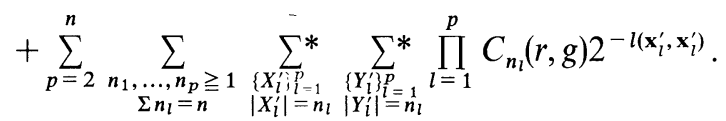

Next we use the following result:

Lemma 5.5. If $\left\{\mathbf{x}_{l}^{\prime}\right\}_{l=1}^{p}$ and $\left\{\mathbf{y}_{l}^{\prime}\right\}_{l=1}^{p}$ are partitions of $\mathbf{x}$ and $\mathbf{y}$ satisfying the link property of Lemma 5.4 then

$$
l(\mathbf{x}, \mathbf{y}) \leqq \sum_{l=1}^{p} l\left(\mathbf{x}_{l}^{\prime}, \mathbf{y}_{l}^{\prime}\right)
$$

Proof. We show that, if $\sigma_{l} \in \mathscr{S}\left(\mathbf{x}_{l}^{\prime}, \mathbf{y}_{l}^{\prime}\right)$ then $\prod_{l=1}^{p} \sigma_{l} \in \mathscr{S}(\mathbf{x}, \mathbf{y})$, where

$$
\left(\prod_{l=1}^{p} \sigma_{l}\right)(\alpha, x)=\sigma_{l}(\alpha, x) \quad \text { if } \quad(\alpha, x) \in \mathbf{x}_{l}^{\prime} .
$$

If a block $B_{s}$ contains points of $\mathbf{x} \cup \mathbf{y}$ but not all, then there must exist $k, k^{\prime}$ such that $z_{k} \in B_{s}, z_{k^{\prime}} \notin B_{s}$. Suppose $\mathbf{x}_{k} \cap \mathbf{x}_{l}^{\prime} \neq \emptyset$ and $\mathbf{y}_{k^{\prime}} \cap \mathbf{y}_{l}^{\prime} \neq \emptyset$. Then $B_{s}$ contains a point (namely $z_{k}$ ) of $\mathbf{x}_{l}^{\prime}$ but not all points of $\mathbf{y}_{l}^{\prime}$. Therefore $B_{s}$ is connected via $\sigma_{l}$ to another block $B_{s}^{\prime}$.

Inserting this into the estimate for $\varrho^{T}$ gives (5.4) with

$$
C_{n}(r, g)=\tilde{C}_{m}(r, g)+\sum_{p=2}^{n} \sum_{\substack{n_{1}, \ldots, n_{p} \geqq 1 \\ \Sigma n_{l}=n}}\left(\frac{n !}{n_{1} ! \ldots n_{p} !}\right)^{2} \prod_{l=1}^{p} C_{n_{l}}(r, g)
$$

depending only on $n$ and $\mathscr{T}(r, g)$. This provides the induction step in the proof of the theorem, proving the estimate for $n$ given Lemma 5.3 and the same estimate for smaller $n$.

$$
\begin{array}{r}
\text { Given } F=\prod_{k=1}^{m} F_{k} \in \mathscr{F} \text { with } \operatorname{supp}\left(F_{k}\right)=\left\{z_{k}\right\} \text {, we define } \\
\qquad d_{k}(F)=q_{1}\left(F_{k}\right)+q_{2}\left(F_{k}\right)(\bmod 2) .
\end{array}
$$


Essential in the proof of Lemma 5.3 is the following iterative evaluation of $l(\mathbf{x}, \mathbf{y})$ : Lemma 5.6. Let $\mathbf{x}=\left\{x_{1}, \ldots, x_{n}\right\}$ and $\mathbf{y}=\left\{y_{1}, \ldots, y_{n}\right\}$ and assume that $m^{\prime} \geqq 2$ where

$$
m^{\prime}=\#\left\{z \mid \exists i: \dot{x}_{i}=z \text { or } \dot{y}_{i}=z\right\} .
$$

Assume also that $\left|q_{\alpha}(B)\right| \leqq 1$ for every level-1 block $B$. Then

$$
l(\mathbf{x}, \mathbf{y})=l(\dot{\mathbf{x}}, \dot{\mathbf{y}})+m^{\prime}-\frac{1}{2} \sum_{k=1}^{m^{\prime}} d_{k}^{\prime} .
$$

Here $\dot{\mathbf{x}}=\left(\dot{x}_{i}\right)_{i=1}^{n}, \dot{\mathbf{y}}=\left(\dot{y}_{i}\right)_{i=1}^{n}$, and

$$
d_{k}^{\prime}=d_{k}\left(\psi_{\dot{\mathbf{x}}} \bar{\psi}_{\mathbf{y}}\right)=\#\left\{i \mid \dot{x}_{i}=z_{k}\right\}-\#\left\{i \mid \dot{y}_{i}=z_{k}\right\}(\bmod 2) .
$$

Proof. It is clear that if $\sigma \in \mathscr{S}(\mathbf{x}, \mathbf{y})$ then $\tilde{\sigma} \in \mathscr{S}(\dot{\mathbf{x}}, \dot{\mathbf{y}})$, where $\tilde{\sigma}\left(\dot{x}_{i}\right)=\sigma\left(x_{i}\right)$. On the other hand, if $\tilde{\tau} \in \mathscr{S}(\dot{\mathbf{x}}, \dot{\mathbf{y}})$ then we can modify $\tau$ to $\sigma \in \mathscr{S}(\mathbf{x}, \mathbf{y})$ so that $l(\sigma)=l(\tau)$ and $l(\tilde{\sigma})$ $=l(\tilde{\tau})$. (See the remark in the proof of Lemma 5.2.)

Next we use Lemma 5.2 and the fact that $\left|q_{\alpha}\left(B\left(z_{k}\right)\right)\right| \leqq 1$ for each $k$ to conclude that we have the following possibilities for each block: $B\left(z_{k}\right) \cap(\mathbf{x} \cup \mathbf{y})=\left\{x_{i}\right\},\left\{x_{i}, x_{j}\right\}$, $\left\{y_{i}\right\},\left\{y_{i}, y_{j}\right\}$ or $\left\{x_{i}, y_{j}\right\}$. The result now follows from the fact that $l(\mathbf{x}, \mathbf{y})-l(\dot{\mathbf{x}}, \dot{\mathbf{y}})$ equals the number of blocks containing a point $x_{i}$.

Proof of Lemma 5.3. Denote the points of support of the successive $F^{(p)}=T^{p} F$ by $z_{k}^{(p)}$, that is $F^{(p)}=\prod_{k=1}^{m_{p}} F_{k}^{(p)}$ with $\operatorname{supp}\left(F_{k}^{(p)}\right)=\left\{z_{k}^{(p)}\right\}$. We are going to proceed by induction on the number $r$ of $p$ 's such that $m_{p}>m_{p+1} \geqq 1$. This number is obviously less than $m$. Clearly, if $r=0$ then $l(F)=0$ and $m=1$ and

$$
|\varrho(; F)|=|\varrho(F)| \leqq\left|f_{0}\right|+\left(\left|f_{1}\right|+\left|f_{2}\right|\right) A_{2}+\left|f_{3}\right| A_{4},
$$

where $F=f_{0}+f_{1} \psi_{1} \bar{\psi}_{1}+f_{2} \psi_{2} \bar{\psi}_{2}+f_{3} \psi_{1} \bar{\psi}_{1} \psi_{2} \bar{\psi}_{2}$ and $A_{2}(r, g)$ and $A_{4}(r, g)$ are constants bounding $\varrho\left(\psi_{1} \bar{\psi}_{1}\right)=\varrho\left(\psi_{2} \bar{\psi}_{2}\right)$ and $\varrho\left(\psi_{1} \bar{\psi}_{1} \psi_{2} \bar{\psi}_{2}\right)$ respectively on the trajectory $\mathscr{T}(r, g)$.

Now consider the induction step. We want to reduce $r$ by 1 so we consider $p$ such that $m=m_{p}>m_{p+1} \geqq 1$. Now, if $m^{\prime}=m$ then $\varrho\left(\prod_{k=1}^{m} ; F_{k}\right)=\varrho^{\prime}\left(\prod_{k=1}^{m} ; T F_{k}\right)$ and in $T F_{k}$ we can omit a constant term. Iterating we can write

$$
\varrho(; F)=2^{-p m+p_{k=1}^{m} d_{k} / 2} \varrho^{(p)}\left(\prod_{k=1}^{m} ; \widetilde{F}_{k}^{(p)}\right),
$$

where $\widetilde{F}_{k}^{(p)}=\widetilde{T}^{p} F_{k}$ and $\widetilde{T}=2^{1-d_{k}^{\prime} / 2}\left(1-P_{0}\right) T\left(1-P_{0}\right)$ and $P_{0}$ is the projection on the constants in the class $(0,0)$. The transformation $\tilde{T}$ is given by a matrix $\tilde{M}$ which decomposes into matrices $\tilde{M}_{q_{1}, q_{2}}$ for each class $\left(q_{1}, q_{2}\right)$. Presently we prove:

Lemma 5.7. Let $(r, g)$ be a point in the lower half-plane such that $R^{n}(r, g) \rightarrow\left( \pm \infty, g_{\infty}\right)$. If

$\tilde{M}^{(p)}=\tilde{M}\left(R^{p}(r, g)\right)$
then $\left\|\prod_{p=0}^{q} \tilde{M}^{(p)}\right\|$ is bounded on the trajectory $\mathscr{T}(r, g)$ by a constant $K(r, g)$ independent of $q$.

From Lemma 5.6 it follows that

$$
p m+\frac{1}{2} p \sum_{k=1}^{m} d_{k}=l(F)-l\left(\widetilde{F}^{(p)}\right)
$$


(Notice that we can choose a representative monomial in each $F_{k}$ with $\left|q_{k}\right| \leqq 1$.) Hence

$$
\varrho(; F)=2^{-l(F)+l\left(\tilde{F}^{(p)}\right)} \varrho^{(p)}\left(; \tilde{F}^{(p)}\right),
$$

where

$$
\prod_{k=1}^{m}\left\|\tilde{F}_{k}^{(p)}\right\| \leqq K(r, g)^{m} \prod_{k=1}^{m}\left\|F_{k}\right\|
$$

It remains to prove that

$$
\left|\varrho^{(p)}\left(; \widetilde{F}^{(p)}\right)\right| \leqq 2^{-l\left(\tilde{F}^{(p)}\right)} \widetilde{C}_{m}(r, g) \prod_{k=1}^{m}\left\|\widetilde{F}_{k}^{(p)}\right\|
$$

when it is given that

$$
\left|\varrho^{(p+1)}(; G)\right| \leqq \widetilde{C}_{m(G)}(r, g) \prod_{k=1}^{m(G)}\left\|G_{k}\right\| 2^{-l(G)}
$$

for all $G=\prod_{k=1}^{m(G)} G_{k} \in \mathscr{F}$ with $m(G)<m_{p}$.

Without loss of generality we can put $p=0$ here. From (5.20) it follows that

$$
\left|\varrho\left(\prod_{k=1}^{m^{\prime}} ; \prod_{l: z_{l}=z_{k}^{\prime}} F_{l}\right)\right| \leqq \tilde{K}(r, g)^{m^{\prime}} \tilde{C}_{m^{\prime}}(r, g) \prod_{l=1}^{m}\left\|F_{l}\right\| 2^{-l\left(F^{\prime}\right)}
$$

for all $F=\prod_{k=1}^{m^{\prime}} \prod_{l: z_{l}=z_{k}^{\prime}} F_{l} \in \mathscr{F}$ and $m^{\prime}<m$, where $\tilde{K}(r, g)$ is a constant depending only on $\mathscr{T}(r, g)$. Let us first remark that it is sufficient to prove (5.19) for a monomial $F=\prod_{l=1}^{m} \mathbf{e}_{v_{l}}\left(z_{l}\right)$, where $\mathbf{e}_{v}(v=1, \ldots, 15)$ denote the monomials of Table 5.1. We proceed as below Lemma 5.4 using the following analogue of Lemma 5.4:

$$
\varrho\left(\prod_{k=1}^{m^{\prime}} ; \prod_{l: z_{l}=z_{k}^{\prime}} \mathbf{e}_{v_{l}}\left(z_{l}\right)\right)=\prod_{\left\{\Lambda_{l}\right\}}^{*}(-1)^{\pi\left(\left\{\Lambda_{l}\right\}\right)} \prod_{i} \varrho\left(\prod_{l \in \Lambda_{l}} ; \mathbf{e}_{v_{l}}\left(z_{l}\right)\right),
$$

where the sum is over all partitions $\left\{\Lambda_{i}\right\}$ of $\{1, \ldots, m\}$ such that every pair $\left(k, k^{\prime}\right)$ is connected by a chain of "links" $\left(k_{1}, k_{2}\right), \ldots,\left(k_{r}, k_{r+1}\right)$. A link $\left(k, k^{\prime}\right)$ satisfies:

$$
\exists i:\left(\exists l \in \Lambda_{i}: \dot{z}_{l}=z_{k}^{\prime} \text { and } \exists l^{\prime} \in \Lambda_{i}: \dot{z}_{l^{\prime}}=z_{k^{\prime}}^{\prime}\right) \text {. }
$$

$\pi\left(\left\{\Lambda_{i}\right\}\right)$ is the number of odd transpositions needed to reorder $\{1, \ldots, m\}$ according to $\left\{\Lambda_{i}\right\}$ [assuming that the $\mathbf{e}_{v_{l}}$ in the left-hand side of (5.22) are in increasing order]. The required result now follows by moving all terms in the sum with $\Lambda_{i} \neq\{1, \ldots, m\}$ to the left and using induction on $n$ and Lemma 5.5. Notice that the number of terms in the sum is bounded by $m^{m+1}$ and that $m \leqq n \leqq 4 m$.

Proof of Lemma 5.7. The classes of Table 5.1 transform according to the matrices $\tilde{M}_{q_{1}, q_{2}}$ given by

$$
\begin{gathered}
\left.\tilde{M}_{-1,-1}=\tilde{M}_{+1,+1}=1 \quad \text { [i.e. } T\left(\psi_{1} \psi_{2}\right)=2^{-1} \psi_{1}^{\prime} \psi_{2}^{\prime}\right] \\
\tilde{M}_{-1,0}=\tilde{M}_{0,-1}=\tilde{M}_{+1,0}=\tilde{M}_{0,+1}=\left(\begin{array}{cc}
1 & 0 \\
c_{1} & 2 g\left(c_{1}^{2}-c_{2}\right)
\end{array}\right), \\
\tilde{M}_{0,0}=2 A^{(0)}
\end{gathered}
$$


where $A^{(0)}$ is the matrix defined in (4.18), and

$$
\tilde{M}_{-1,+1}=\tilde{M}_{+1,-1}=\frac{1}{2}\left(1-4 g c_{2}\right) \text {. }
$$

Now, as $(r, g) \rightarrow(0,0), \tilde{M}_{-1,0} \rightarrow\left(\begin{array}{cc}1 & 0 \\ 1 / 4 & 0\end{array}\right)$ and

$$
\tilde{M}_{0,0} \rightarrow\left(\begin{array}{ccc}
1 & 0 & 0 \\
0 & 1 & 0 \\
1 / 4 & 1 / 4 & 1 / 2
\end{array}\right)
$$

and $\tilde{M}_{-1,+1} \rightarrow \frac{1}{2}$, all exponentially fast. Furthermore,

and

$$
\left(\begin{array}{cc}
1 & 0 \\
1 / 4 & 0
\end{array}\right)^{n}=\left(\begin{array}{cc}
1 & 0 \\
1 / 4 & 0
\end{array}\right)
$$

$$
\left(\begin{array}{ccc}
1 & 0 & 0 \\
0 & 1 & 0 \\
1 / 4 & 1 / 4 & 1 / 2
\end{array}\right)^{n}=\left(\begin{array}{ccc}
1 & 0 & 0 \\
0 & 1 & 0 \\
\frac{1}{2}\left(1-2^{-n}\right) & \frac{1}{2}\left(1-2^{-n}\right) & \frac{1}{2}
\end{array}\right)
$$

are both bounded. The bound on the norm of $\prod_{p=0}^{q} \tilde{M}^{(p)}$ then follows from the trivial Lemma 5.8. Let $M^{(n)}$ be a sequence of matrices satisfying $M^{(n)}=M_{\infty}+\gamma^{-n} E^{(n)}$, where $\left\|M_{\infty}^{n}\right\| \leqq A_{0},\left\|E^{(n)}\right\| \leqq c$, and $\gamma>1$. Then $\left\|\prod_{k=0}^{p} M^{(k)}\right\| \leqq A_{0} e^{\gamma c /(\gamma-1)}$.

As $(r, g) \rightarrow\left( \pm \infty, g_{\infty}\right)$,

$$
\tilde{M}_{-1,0} \rightarrow\left(\begin{array}{ll}
1 & 0 \\
0 & 0
\end{array}\right), \quad \tilde{M}_{0,0} \rightarrow\left(\begin{array}{ccc}
1 & 0 & 0 \\
0 & 1 & 0 \\
0 & 0 & 1 / 2
\end{array}\right), \quad \text { and } \quad \tilde{M}_{-1,+1} .
$$

This proves Lemma 5.7 for all trajectories except the trajectory going off to $\left(-\frac{2}{3},-\infty\right)$. For the latter we remark that as $(r, g) \rightarrow\left(-\frac{2}{3},-\infty\right)$,

$$
\tilde{M}_{-1,0} \rightarrow\left(\begin{array}{cc}
1 & 0 \\
0 & 1 / 2
\end{array}\right), \quad \tilde{M}_{0,0} \rightarrow\left(\begin{array}{ccc}
2 & 0 & -8 / 3 \\
0 & 2 & -8 / 3 \\
0 & 0 & 2
\end{array}\right), \quad \text { and } \tilde{M}_{-1,+1} \rightarrow 1,
$$

so that the bound on the product of matrices $\tilde{M}^{(p)}$ does not hold.

If we extend the definition of $s(x, y)$ to $n$-tuples by

$$
s(\mathbf{x}, \mathbf{y})=\max _{\sigma \in \mathscr{S}_{n}} \sum_{i=1}^{n} s\left(x_{i}, y_{\sigma(i)}\right),
$$

then we have the following

Corollary. Let $(r, g)$ be a point in the lower half-plane $(g<0)$ such that $R^{n}(r, g)$ $\rightarrow\left( \pm \infty, g_{\infty}\right)$. For all $n \geqq 1$ there exists a constant $D_{n}(r, g)$ depending only on $n$ and the trajectory $\mathscr{T}(r, g)$ such that

$$
\left|\varrho\left(\psi_{\alpha_{1} x_{1}} \bar{\psi}_{\beta_{1} y_{1}} \ldots \psi_{\alpha_{n} x_{n}} \bar{\psi}_{\beta_{n} y_{n}}\right)\right| \leqq D_{n}(r, g) 2^{-s\left(x_{1}, \ldots, x_{n} ; y_{1}, \ldots, y_{n}\right)}
$$

for any set of $2 n$ points $x_{1}, \ldots, x_{n}, y_{1}, \ldots, y_{n}$ and indices $\alpha_{1}, \ldots, \alpha_{n}, \beta_{1}, \ldots, \beta_{n} \in\{1,2\}$. 
Proof. Use the formula (5.2) together with the relation

$$
s(\mathbf{x}, \mathbf{y}) \leqq \sum_{k=1}^{m} l\left(\mathbf{x}_{k}, \mathbf{y}_{k}\right)
$$

Remark. This bound on the $n$-point functions is also correct in the case of the "critical" trajectories $\mathscr{T}\left(r_{s}(g), g\right)$.

\section{The Continuum Limit}

Using the information about the decay of correlation functions obtained in Sect. 5 we shall prove the existence of the continuum limit for theories corresponding to points $(r, g)$ on the trajectories receding from the "trivial" fixed point $(0,0)$. This is particularly easy for the unstable line $g=0, r \geqq 0$ : these theories are "Gaussian" so that we need only consider the 2-point functions. The theories with $g<0$ are nontrivial and we have to consider the behaviour of the general $n$-point function. However, it turns out that the result of the Corollary of Theorem 5 is strong enough to ensure the existence of the continuum limit, so that the latter also exists for the theories with $R^{n}(r, g) \rightarrow\left(-\frac{2}{3},-\infty\right)$. The existence of an unstable line for the fixed point $\left(-\frac{4}{3}, \frac{8}{27}\right)$ suggests that there also exist continuum limits associated with this fixed point. In fact this appears not to be the case in the proper sense to be defined below. This pathology can be seen to be connected to the fact that the thermodynamic limit does not exist for this fixed point.

The usual way to proceed in constructing a continuum limit is the following. We choose a sequence $\left(r_{n}, g_{n}\right)$ converging to a point on either of the two critical lines such that $\mathscr{R}^{n} v_{m+n}$ converges as $n \rightarrow \infty$ for all $m$ (large enough). Here $v_{n}=v\left(r_{n}, g_{n}\right)$ is the potential with coupling constants $\left(r_{n}, g_{n}\right)$ defined by $(2.8)$ and $\mathscr{R} v_{n}=v\left(R\left(r_{n}, g_{n}\right)\right)$ is the transformed potential. Thus we obtain states $\varrho_{m}$ satisfying

$$
\mathscr{R} \varrho_{m}=\varrho_{m-1} .
$$

We then define states $\tilde{\varrho}_{m}$ "living" on the rescaled lattices $2^{-m} \mathbb{Z}^{2}$ by

$$
\tilde{\varrho}_{m}(F)=\varrho_{m}\left(F\left(2^{m / 2} \psi_{2 m} *, 2^{m / 2} \bar{\psi}_{2 . m} *\right)\right),
$$

where $F\left(\psi_{m}, \bar{\psi}_{m}\right)$ is a polynomial in the fields $\psi_{m}$ living on $2^{-m} \mathbb{Z}^{2}$. By (6.1) we have

$$
\tilde{\varrho}_{m-1}(F)=\tilde{\varrho}_{m}\left(F\left(\frac{1}{4} \sum_{x \in B(0)} \psi_{m}\left(\cdot+2^{-m} x\right), \frac{1}{4} \sum_{x \in B(0)} \bar{\psi}_{m}\left(\cdot+2^{-m} x\right)\right)\right) \text {. }
$$

We want to consider the fields $\psi_{m}$ as the means of a putative continuum field $\psi$ over blocks $\square_{m}$ :

$$
\psi_{m}(\underline{x})=2^{2 m} \psi\left(1_{\square_{m}(x)}\right) \quad\left(x \in 2^{-m} \mathbb{Z}^{2}\right),
$$

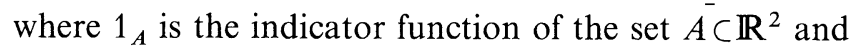

$$
\square_{m}(\underline{x})=\left\{\underline{v} \in \mathbb{R}^{2} \mid \underline{x}_{i}-2^{-m-1} \leqq \underline{v}_{i} \leqq \underline{x}_{i}+2^{-m-1}\right\} .
$$

Approximating the smooth functions $f_{i}$ and $g_{i}(i=1, \ldots, k)$ on $\mathbb{R}^{2}$ successively by

$$
f_{i}^{(m)}=\sum_{x \in 2^{-m} \mathbb{Z}^{2}} f_{i}(\underline{x}) 1_{\square m(\underline{x})}
$$


and $g_{i}^{(m)}$ defined analogously, we accordingly define the $k$-point function of the continuum state $\varrho$ on $\mathscr{G}(E)$ with $E=S\left(\mathbb{R}^{2}, \mathbb{R}\right)$ by

$$
\begin{aligned}
\tilde{\varrho}\left(\psi_{\alpha_{1}}\left(f_{1}\right) \bar{\psi}_{\beta_{1}}\left(g_{1}\right) \ldots \psi_{\alpha_{k}}\left(f_{k}\right) \bar{\psi}_{\beta_{k}}\left(g_{k}\right)\right) \\
=\lim _{m \rightarrow \infty} \int d_{m} \underline{x}_{1} \ldots \int d_{m} \underline{x}_{k} \int d_{m} \underline{y}_{1} \ldots \int d_{m} \underline{y}_{k} f_{1}\left(\underline{x}_{1}\right) g_{1}\left(\underline{y}_{1}\right) \ldots f_{k}\left(\underline{x}_{k}\right) g_{k}\left(\underline{y}_{k}\right) \\
\quad \times 2^{m k} \varrho_{m}\left(\psi_{\alpha_{1}, 2^{m} x_{1}} \bar{\psi}_{\beta_{1}, 2^{m} \underline{y}_{1}} \ldots \psi_{\alpha_{1}, 2^{m} \underline{x}_{k}} \bar{\psi}_{\beta_{k}, 2^{m} \underline{y}_{k}}\right) .
\end{aligned}
$$

Here $\int d_{m} \underline{x} \equiv 2^{-2 m} \sum_{x \in 2^{-m} \mathbb{Z}^{2}}$ and $f_{1}, \ldots, f_{k}, g_{1}, \ldots, g_{k} \in S\left(\mathbb{R}^{2} ; \mathbb{R}\right)$. One easily checks with the help of (6.3) that the limit (6.7) would be trivial, if $f_{i}=f_{i}^{(m)}$ and $g_{i}=g_{i}^{(m)}$ for some $m$ and all $i$. We remark that the above procedure is only possible in the infinite volume limit. Hence we must take $\left(r_{n}, g_{n}\right) \in \mathscr{D}$ for all $n$. As the transformation $R$ does not depend on $N$ it is unchanged in the infinite volume limit. From Lemma 7.1 below we can deduce that, if we keep $g_{n}=g_{0} \geqq 0$ fixed and let $r \downarrow r_{c}\left(g_{0}\right)$ then we end up with a Gaussian theory, i.e. $\varrho_{m}=\mathscr{R}^{-m} \varrho_{0}$, where $\varrho_{0}$ is given by a point $\left(r_{0}, 0\right)$ with $r_{0}>0$. [If we take $r_{n}=r_{c}\left(g_{0}\right)$ then $r_{0}=0$.] This means that we might as well start from $\varrho_{m}=\mathscr{R}^{-m} \varrho_{0}$. In the same way, taking $r_{n}=r_{0}$ fixed and letting $g_{n} \uparrow g_{c}\left(r_{0}\right)$ from below we obtain points $\left(\tilde{r}_{m}, \tilde{g}_{m}\right)$ on the unstable line of the fixed point $\left(-\frac{4}{3}, \frac{8}{27}\right)$ tending to this fixed point as $m \rightarrow \infty$. Finally, we can take sequences $\left(r_{n}, g_{n}\right)$ $=R^{-n}\left(r_{0}, g_{0}\right)$ converging to $(0,0)$ from the lower half-plane, i.e. with $g_{0}<0$. In that case $\left(\tilde{r}_{m}, \tilde{g}_{m}\right)=R^{-m}\left(r_{0}, g_{0}\right)$. It remains to verify that the limit (6.7) exists in these three cases. In fact this is true only in the cases where $\left(\tilde{r}_{m}, \tilde{g}_{m}\right) \rightarrow(0,0)$ :

Theorem 6. Let $\varrho_{m}$ be the state $\mathscr{G}\left(E_{\infty}\right)$ with $E_{\infty}=\left(\mathbb{R}^{2}\right)^{\mathbb{Z}^{2}}$ defined by Theorem 4 with $\left(r_{m}, g_{m}\right)=R^{-m}\left(r_{0}, g_{0}\right)$, where $g_{0}<0$ or $g_{0}=0$ and $r_{0} \geqq 0$. Then the continuum limit (6.7) exists for all $k$ and $f_{1}, \ldots, f_{k}, g_{1}, \ldots, g_{k} \in S\left(\mathbb{R}^{2} ; \mathbb{R}\right)$.

Proof. We estimate the difference between the $m$-th and the $n$-th element in the sequence (6.7) with $n>m$, and rewrite the "integrals" $\int d_{m} \underline{x}_{i}$ as "integrals" $\int d_{n} \underline{x}_{i}$ by replacing $f_{i}$ with $f_{i}^{(m)}$.

Now, given $\varepsilon>0$ we can choose $m$ so large that

$$
\left|f_{i}(\underline{x})-f_{i}^{(m)}(\underline{x})\right|<\varepsilon\left(1+|\underline{x}|^{2}\right)^{-2} \quad \forall \underline{x} \in \mathbb{R}^{2}, i=1, \ldots, k
$$

and the same for $g_{i}(i=1, \ldots, k)$. Assume also that we have the bound

$$
\left|f_{i}(\underline{x})\right| \leqq M\left(1+|\underline{x}|^{2}\right)^{-2} \quad \forall \underline{x} \in \mathbb{R}^{2}, i=1, \ldots, k,
$$

and the same for $g_{i}(i=1, \ldots, k)$. Then we find

$$
\begin{aligned}
& \mid \int d_{n} \underline{x}_{1} \ldots \int d_{n} \underline{x}_{k} \int d_{n} \underline{y}_{1} \ldots \int d_{n} \underline{y}_{k} \\
& \times\left\{f_{1}\left(\underline{x}_{1}\right) g_{1}\left(\underline{y}_{1}\right) \ldots f_{k}\left(\underline{x}_{k}\right) g_{k}\left(\underline{y}_{k}\right)-f_{1}^{(m)}\left(\underline{x}_{1}\right) g_{1}^{(m)}\left(\underline{y}_{1}\right) \ldots f_{1}^{(m)}\left(\underline{x}_{k}\right) g_{1}^{(m)}\left(\underline{y}_{k}\right)\right\} \\
& \times 2^{n k} \varrho_{n}\left(\psi_{\alpha_{1}, 2^{n} x_{1}} \bar{\psi}_{\beta_{1}, 2^{n} \underline{y}_{1}} \ldots \psi_{\alpha_{k}, 2^{n} x_{k}} \bar{\psi}_{\beta_{k}, 2^{n} \underline{y}_{k}}\right) \mid \\
& \leqq 2 k M^{2 k-1} \varepsilon \int d_{n} \underline{x}_{1} \ldots \int d_{n} \underline{x}_{k} \int d_{n} \underline{y}_{1} \ldots \int d_{n} \underline{y}_{k} \\
& \times \prod_{i=1}^{k}\left(1+\left|\underline{x}_{i}\right|^{2}\right)^{-2}\left(1+\left|\underline{y}_{i}\right|^{2}\right)^{-2} 2^{n k}\left|\varrho_{n}\left(\psi_{\alpha_{1}, 2^{n} x_{1}} \bar{\psi}_{\beta_{1}, 2^{n} \underline{y}_{1}} \ldots \psi_{\alpha_{k}, 2^{n_{x}}} \bar{\psi}_{\beta_{k}, 2^{n} \underline{y}_{k}}\right)\right| \text {, }
\end{aligned}
$$

where by the Corollary of Theorem 6 ,

$$
\left|\varrho_{n}\left(\psi_{\alpha_{1}, 2^{n} x_{1}} \bar{\psi}_{\beta_{1}, 2^{n_{y_{1}}}} \ldots \psi_{\alpha_{k}, 2^{n} \underline{x}_{k}} \bar{\psi}_{\beta_{k}, 2^{n_{\underline{n}}} \underline{y}_{k}}\right)\right| \leqq D_{k}(r, g) 2^{-s(\mathbf{x}, \mathbf{y})}
$$

with $x_{i}=2^{n} \underline{x}_{i}$ and $y_{i}=2^{n} \underline{y}_{i}$. We now use the following simple estimate: 
Lemma 6.1. For $x, y \in \mathbb{Z}^{2}$ the following bound holds:

$$
2^{-s(x, y)} \leqq \frac{3}{1+|x-y|} .
$$

It follows from this lemma that

$$
2^{-s(\mathbf{x}, \mathbf{y})} \leqq \max _{\sigma \in \mathscr{S}_{k}} \prod_{i=1}^{k} \frac{3}{1+2^{n}|\underline{x}-\underline{y}|},
$$

and hence the right-hand expression of (6.8) is bounded by

$$
2 k M^{2 k-1} k ! D_{k}(r, g) \varepsilon\left\{\int d_{n} \underline{x} \int d_{n} \underline{y}\left(1+|\underline{x}|^{2}\right)^{-2}\left(1+|\underline{y}|^{2}\right)^{-2} \frac{3 \cdot 2^{n}}{1+2^{n}|\underline{x}-\underline{y}|}\right\}^{k} .
$$

The double "integral" is bounded by a constant independent of $n$. This concludes the proof of the existence of the continuum limit.

Notice that in the case that $\left(\tilde{r}_{m}, \tilde{g}_{m}\right)=R^{-m}\left(r_{0}, g_{0}\right) \rightarrow\left(-\frac{4}{3}, \frac{8}{27}\right)$ we have $\varrho_{n}\left(\psi_{\alpha x} \bar{\psi}_{\beta y}\right)$ $\approx \delta_{\alpha \beta} \frac{\lambda_{+}^{n}}{1+|x-y|}$, with $\lambda_{+}=\frac{35}{8}+\frac{3}{8} \sqrt{137}$, if $s(x, y) \ll 2^{n}$. This means that the continuum limit does not exist for the same reason that the infinite volume limit does not exist at the fixed point.

\section{Proofs of the Results in Sect. 3}

The existence part of Theorem 1 follows from

Lemma 7.1. Let $\gamma$ be a continuous curve in $\mathscr{S}\left(g_{0}\right)$ with endpoints $\left(r_{1}, g_{1}\right)$ and $\left(r_{2}, g_{2}\right)$ at the left- and right-hand boundary respectively, i.e. $r_{1}=\max \left\{r_{-}\left(g_{1}\right), r_{0}\left(g_{1}\right)\right\}$ and $r_{2}=r_{+}\left(g_{2}\right)$. Then $\gamma^{\prime}=R(\gamma)$ is contained within the region $0 \leqq g \leqq g_{0}^{\prime}=F_{+}\left(g_{0}\right)$, where the function $F_{+}$is defined by

$$
F_{+}(g)=\frac{g}{\left(1+r_{+}(g) / 2\right)^{2}} .
$$

Furthermore, the endpoints $\left(r_{1}^{\prime}, g_{1}^{\prime}\right)$ and $\left(r_{2}^{\prime}, g_{2}^{\prime}\right)$ satisfy

$$
r_{1}^{\prime} \leqq \max \left\{r_{-}\left(g_{1}^{\prime}\right), r_{0}\left(g_{1}^{\prime}\right)\right\} \quad \text { and } \quad r_{2}^{\prime} \geqq r_{+}\left(g_{2}^{\prime}\right) .
$$

Proof. If $(r, g)$ is a point of $\gamma$ then $g \leqq 2(1+r)^{2}$, so $\frac{\partial}{\partial r}\left\{\frac{(1+r)^{2}-g / 2}{(1+r)^{2}-g / 4}\right\} \geqq 0$. Consequently,

$$
g^{\prime} \leqq g\left\{\frac{\left(1+r_{+}(g)\right)^{2}-g / 2}{\left(1+r_{+}(g)\right)^{2}-g / 4}\right\}^{2}=F_{+}(g) \leqq F_{+}\left(g_{0}\right)=g_{0}^{\prime} .
$$

This proves the first statement of the lemma. The second statement follows by a simple calculation.

The existence of the critical line now follows with the interval argument of Sinai and Bleher [3]:

Proposition 7.1. For all $g_{0}>0$ there exists a critical $r$-value $r_{c}\left(g_{0}\right)$ such that $R^{n}\left(r_{c}\left(g_{0}\right), g_{0}\right) \rightarrow(0,0)$ as $n \rightarrow \infty$. 
Proof. Let $I_{0}$ be the interval of $r$-values: $I_{0}=\left[\max \left\{r_{-}\left(g_{0}\right), r_{0}\left(g_{0}\right)\right\}, r_{+}\left(g_{0}\right)\right]$, and let $\gamma_{0}$ be the curve of points $\left(r, g_{0}\right)$ where $r$ runs through $I_{0}$. Let $\gamma_{1}$ be a maximal connected part of $\gamma_{0}^{\prime} \cap \mathscr{S}\left(g_{0}^{\prime}\right)$ and let $I_{1} \subset I_{0}$ be such that the corresponding part of $\gamma_{0}$ is mapped onto $\gamma_{1}$. Proceeding in this way we find $I_{n} \subset I_{n-1}$ such that $R^{n}$ maps the corresponding part of $\gamma_{0}$ onto the connected part $\gamma_{n}$ of $\gamma_{n-1}^{\prime} \cap \mathscr{S}\left(g_{n}\right)$ with $g_{n}=F_{+}\left(g_{n-1}\right)$. We take $r_{c}\left(g_{0}\right) \in \bigcap_{n \geq 0} I_{n}$. Then, by construction, $R^{n}\left(r_{c}\left(g_{0}\right), g_{0}\right) \in \mathscr{S}\left(g_{n}\right)$. The fact that $F_{+}^{n}\left(g_{0}\right) \rightarrow 0$ implies that $R^{n}\left(r_{c}\left(g_{0}\right), g_{0}\right) \rightarrow(0,0)$.

The proof of the uniqueness is rather delicate. Again we make use of a horizontal curve $\gamma$ and prove by induction that it is stretched in the $r$-direction. The induction works only after the first iteration with which we have to deal separately. Let us first define the variable

$$
z=\frac{g}{4(1+r)^{2}} .
$$

Then $1+r=\frac{1-z}{1-2 z}\left(1+\frac{1}{2} r^{\prime}\right)$ and $z^{\prime}=z\left(\frac{1+r^{\prime} / 2}{1+r^{\prime}}\right)^{2}$. The first iteration step is dealt with in the following two lemmas. We omit the proofs which are straightforward though somewhat tedious.

Lemma 7.2. If $(r, g) \in \mathscr{S}\left(g_{0}\right)$ for some $g_{0}>0$, and $\left(r^{\prime}, g^{\prime}\right) \in \mathscr{S}\left(g_{0}\right)$ then $g^{\prime} \leqq 2\left(1+\frac{1}{2} r^{\prime}\right)^{2}$ and $r^{\prime} \leqq \frac{2}{\sqrt{3}}$.

Lemma 7.3. Assume $(r, g) \in \mathscr{S}\left(g_{0}\right)$ and $\left(r^{\prime}, g^{\prime}\right) \in \mathscr{S}\left(g_{0}\right)$. Then $0 \leqq \frac{\partial g^{\prime}}{\partial r}<6 z^{\prime}\left(1+r^{\prime}\right) \frac{\partial r^{\prime}}{\partial r}$.

For the next iterations we may assume that $r<\frac{2}{\sqrt{3}}$ and $g \leqq 2\left(1+\frac{1}{2} r\right)^{2}<\frac{8}{3}+\frac{4}{3} \sqrt{3}$.

But then $g^{\prime} \leqq F_{+}\left(\frac{8}{3}+\frac{4}{3} \sqrt{3}\right)=2$. In addition $g \leqq 2\left(1+\frac{1}{2} r\right)^{2} \Rightarrow z \leqq z_{0}$, where $z_{0}$ is given by $4 z_{0}(1+r)^{2}=4 r(1+r)=2\left(1+\frac{1}{2} r\right)^{2}$, i.e. $z_{0}=\frac{4 \sqrt{2}-2}{4 \sqrt{2}+5}<\frac{2}{5}$.

Lemma 7.4. Let $\gamma$ be a curve in $\mathscr{S}\left(g_{0}\right)$ lying entirely below the line $g=2\left(1+\frac{1}{2} r\right)^{2}$. Assume that its tangent satisfies

$$
0 \leqq \frac{d g}{d r}<6 z(1+r)
$$

and assume also that the endpoints of $\gamma^{\prime}$ lie within $\mathscr{S}\left(\mathrm{g}_{0}\right)$. Then $0 \leqq \frac{d g^{\prime}}{d r^{\prime}}<6 z^{\prime}\left(1+r^{\prime}\right)$. In particular $\gamma^{\prime}$ lies entirely within $\mathscr{S}\left(g_{0}\right)$.

Proof. Put $\frac{d g}{d r}=4 \alpha z(1+r)$. Then

$$
\frac{\partial r^{\prime}}{\partial r}+\frac{\partial r^{\prime}}{\partial g} \frac{d g}{d r}=2 \frac{1-(1+\alpha) z+2 z^{2}}{(1-z)^{2}}>0 .
$$

Using the fact that $z \leqq \frac{2}{5}$ the result now follows from the formula

$$
\frac{d g^{\prime}}{d r^{\prime}}=\left(\frac{\partial g^{\prime}}{\partial r}+\frac{\partial g^{\prime}}{\partial g} \cdot \frac{d g}{d r}\right)\left(\frac{\partial r^{\prime}}{\partial r}+\frac{\partial r^{\prime}}{\partial g} \cdot \frac{d g}{d r}\right)^{-1}
$$


Proposition 7.2. For all $g_{0}>0$ there exists a unique critical value $r_{c}\left(g_{0}\right)>0$ such that

$$
R^{n}\left(r_{c}\left(g_{0}\right), g_{0}\right) \in \mathscr{S}\left(g_{0}\right) \text { for all } n \geqq 0
$$

and $R^{n}\left(r_{c}\left(g_{0}\right), g_{0}\right) \rightarrow(0,0)$ as $n \rightarrow \infty$.

Proof. Suppose that for a certain value of $g_{0}>0$ there exist two values $r_{1}\left(g_{0}\right)$ and $r_{2}\left(g_{0}\right)$ with the required properties. Consider the horizontal line $\gamma_{0}$ connecting the points $\left(r_{1}\left(g_{0}\right), g_{0}\right)$ and $\left(r_{2}\left(g_{0}\right), g_{0}\right)$, and its iterates $\gamma_{n}$. According to Lemma 7.3, $\gamma_{1} \subset \mathscr{S}\left(g_{0}\right)$ and its tangent is less than $6 z(1+r)$. Furthermore, Lemma 7.2 shows that $(r, g) \in \gamma_{1} \Rightarrow g<2\left(1+\frac{1}{2} r\right)^{2}$. Thus we can use Lemma 7.4 to iterate $R$ and find that (7.3) holds for all $\gamma_{n}$. For large $n, z$ becomes small and from (7.4) we have $\Delta r^{\prime}>\frac{3}{2} \Delta r$, where $\Delta r$ is the distance in the $r$-direction between the endpoints. Clearly, this contradicts the hypothesis that $\gamma_{n}$ lies in $\mathscr{S}\left(g_{0}\right)$ for all $n$.

The proof of Theorem 2 is similar to the above proof of Theorem 1. We simply state the necessary lemmas.

Lemma 7.5. Let $\gamma$ be a continuous curve in $\mathscr{T}_{1}$ with endpoints at the upper-and lower boundary respectively, i.e. assume that the points $\left(r_{1}, g_{1}\right)$ and $\left(r_{2}, g_{2}\right)$ satisfy $g_{1}=g_{-}\left(r_{1}\right)$ and $g_{2}=g_{+}\left(r_{2}\right)$. Then $\gamma^{\prime}$ is contained in the region $2\left(1+\frac{1}{2} r^{\prime}\right)^{2} \leqq g^{\prime}$ $\leqq \frac{8}{3}\left(1+\frac{1}{2} r^{\prime}\right)^{2}$ with $r^{\prime}>-2$, and $g_{1}^{\prime} \leqq g_{+}\left(r_{1}^{\prime}\right)$ and $g_{2}^{\prime} \geqq g_{-}\left(r_{2}^{\prime}\right)$. If, in addition, $\gamma$ lies below the line $g=\left(\frac{8}{3}+\varepsilon\right)\left(1+\frac{1}{2} r\right)^{2}$ then $\gamma^{\prime}$ lies above the line $g^{\prime}=\left(\frac{8}{3}-\frac{1}{4} \varepsilon\right)\left(1+\frac{1}{2} r^{\prime}\right)^{2}$ provided that $\varepsilon \leqq \frac{2}{3}$.

Lemma 7.6. Let $\tilde{\gamma}$ be a continuous curve in $\mathscr{T}_{2}$ with endpoints $\left(r_{1}, g_{1}\right)$ and $\left(r_{2}, g_{2}\right)$ satisfying $g_{1}=g_{+}\left(r_{1}\right)$ and $g_{2}=g_{-}\left(r_{2}\right)$. Then $\tilde{\gamma}^{\prime}$ is contained in the region $\frac{8}{3}\left(1+\frac{1}{2} r^{\prime}\right)^{2}$ $\leqq g^{\prime} \leqq \frac{10}{3}\left(1+\frac{1}{2} r^{\prime}\right)^{2}$ with $r^{\prime}>-2$, and $g_{1}^{\prime} \leqq g_{-}\left(r_{1}^{\prime}\right), g_{2}^{\prime} \geqq g_{+}\left(r_{2}^{\prime}\right)$. If $\tilde{\gamma}$ lies above the line $g=\left(\frac{8}{3}-\varepsilon\right)\left(1+\frac{1}{2} r\right)^{2}$ with $\varepsilon \leqq \frac{2}{3}$ then $\tilde{\gamma}^{\prime}$ lies below $g^{\prime}=\left(\frac{8}{3}+\frac{2}{3} \varepsilon\right)\left(1+\frac{1}{2} r^{\prime}\right)^{2}$.

Proof of Theorem 3. The proof consists of a successive reduction to smaller regions.

The case $g>0, r>r_{c}(g)$ reduces to $g<2 r(1+r)$ which subsequently reduces to the case $g<r(1+r)$ where $r^{\prime}>\frac{4}{3} r$ and $g^{(n)} \rightarrow g_{\infty}(r, g)>0$.

The cases $r \leqq r_{0}, g<g_{c}(r)$ and $r<-1, g<g_{+}(r)$ reduce with the help of Lemmas 7.5 and 7.6 to $r<-1,0<g<2(1+r)^{2}$ which reduces further to the case $r<-2,0<g<(1+r)^{2}$ which is trivial.

For $g<0$ one proves the existence and uniqueness of the line $r_{s}(g)$ in the region $-\frac{2}{3}<r<0,0<g<\frac{4 r(1+r)^{2}}{2+3 r}$ in the same way as the existence and uniqueness of $r_{c}(g)$ and $g_{c}(r)$ was established in the proofs of Theorems 1 and 2. Points outside this region are easily seen to move to $\left( \pm \infty, g_{\infty}\right)$ with $g_{\infty}>-\infty$.

\section{Final Remarks}

Although the model that we have studied in this paper is rather artificial it has the advantage that it can be renormalized easily. The renormalization transformation involves only two coupling parameters $r$ and $g$ and is given by the exact transformation formulas (2.16). Thus many technicalities that appear in other models when studying the asymptotic renormalization group behaviour do not 
occur here. Other simple renormalization group transformations were considered by Nelson and Fisher [10]. Their aim was different, however, and they did not consider the decay of correlations or the existence of a continuum limit.

Also unlike most other models it is possible to make non-perturbative, i.e. global statements about the renormalization group flow. In particular we have seen that there exists, apart from the trivial fixed point $(0,0)$, a non-trivial fixed point $\left(-\frac{4}{3}, \frac{8}{27}\right)$, and we have managed to prove the existence and uniqueness of global critical lines for both fixed points. This is not to say that everything about the flow in parameter space is fully understood. Computer studies show that the behaviour in the region above and between the two critical lines is rather erratic. Iterating the inverse mapping for a small part of the critical line near $(0,0)$ one obtains an array of points that seem to be concentrated on a bundle of curves in this region. (For a picture, see [7].) If this behaviour is genuine all these curves are critical lines for the fixed point $(0,0)$ ! Also, some of the points thus obtained lie in the neighbourhood of $(0,0)$ itself.

Some of the unusual features of the renormalization group flow may be due to the hierarchical structure of the model. However, it cannot be ruled out that similar complications occur also in other, more realistic models. For instance, the recurrence of critical points to the neighbourhood of the fixed point is a possibility to be kept in mind. The large null space of $\Gamma_{0}$ means that there is no Hamiltonian formulation for this model. Hence the concept of a phase transition is unclear but, judging by the behaviour of the correlation functions, the line $r_{s}(g)$ in the lower half-plane behaves as a critical line: The decay of correlation functions for points $(r, g)$ on this line is slower than for other points of the lower half-plane.

Acknowledgements. The author wishes to thank Prof. N. M. Hugenholtz for guidance and stimulating discussions. He also thanks Prof. J. T. Lewis for encouraging him to finish the article. Part of the work was carried out in the context of the research programme of the "Stichting voor Fundamenteel Onderzoek der Materie" (F.O.M.), which is financially supported by the "Nederlandse Organisatic voor Zuiver Wetenschappelijk Onderzoek" (Z.W.O.).

\section{References}

1. Baker, G.A.: Ising model with a scaling interaction. Phys. Rev. B 5, 2622 (1972)

2. Berezin, F.A.: The method of second quantization. New York: Academic Press 1966

3. Bleher, P.M., Sinai, Ya.G.: Investigations of the critical point in models of the type of Dyson's hierarchical model. Commun. Math. Phys. 33, 23 (1973)

4. Bleher, P.M., Sinai, Ya.G.: Critical indices for Dyson's asymptotically hierarchical models. Commun. Math. Phys. 45, 247 (1975)

5. Collet, P., Eckmann, J.-P.: A renormalization group analysis of the hierarchical model in statistical mechanics. Lecture Notes in Physics, vol. 74. Berlin, Heidelberg, New York: Springer 1978

6. Dorlas, T.C.: Renormalization of a hierarchical fermion model in two dimensions. In: Statistical mechanics and field theory, mathematical aspects. Lecture Notes in Physics, vol. 257. Berlin, Heidelberg, New York: Springer 1986

7. Dorlas, T.C.: On some aspects of renormalization group theory and hierarchical models. Thesis, University of Groningen 1987

8. Dyson, F.J.: Existence of a phase transition in a one-dimensional ferromagnet. Commun. Math. Phys. 12, 91 (1969)

9. Feldman, J., Magnen, J., Rivasseau, V., Sénéor, R.: A renormalizable field theory: the massive Gross-Neveu model in two dimensions. Commun. Math. Phys. 103, 67 (1986)

10. Fisher, M.E., Nelson, D.R.: Soluble renormalization groups and scaling fields for lowdimensional Ising systems. Ann. Phys. 91, 226 (1975) 
11. Gawedzki, K., Kupiainen, A.: Renormalization group study of a critical lattice model I: convergence to the line of fixed points. Commun. Math. Phys. 82, 407 (1981)

12. Gawedzki, K., Kupiainen, A.: Renormalization group study of a critical lattice model. II. The correlation functions. Commun. Math. Phys. 83, 469 (1982)

13. Gawedzki, K., Kupiainen, A.: Triviality of $\phi_{4}^{4}$ and all that in a hierarchical model approximation. J. Stat. Phys. 29, 683 (1982)

14. Gawedzki, K., Kupiainen, A.: Gross-Neveu model through convergent perturbation expansions. Commun. Math. Phys. 102, 1 (1985)

15. Gawedzki, K., Kupiainen, A.: Continuum limit of the hierarchical $O(N)$ nonlinear $\sigma$-model. Commun. Math. Phys. 106, 533 (1986)

16. Lanford III, O.E.: Bifurcation of periodic solutions into invariant tori: the work of Ruelle and Takens. In: Nonlinear problems in the physical sciences and biology. Lecture Notes in Mathematics, vol. 322. Berlin, Heidelberg, New York: Springer 1973

17. Marsden, J.E., McCracken, M.: The Hopf bifurcation and its applications. Berlin, Heidelberg, New York: Springer 1976

18. Van Strien, S.J.: Center manifolds are not $C^{\infty}$. Math. Zeitschr. 166, 143 (1977)

19. Wilson, K.G.: Renormalization group and critical phenomena, I, II. Phys. Rev. B 4, 3174 (1971)

Communicated by K. Gawedzki 